This is an Open Access article, distributed under the terms of the Creative Commons Attribution licence (http://creativecommons.org/licenses/by/4.0/), which permits unrestricted re-use, distribution, and reproduction in any medium, provided the original work is properly cited.

doi:10.1017/jfm.2016.653

\title{
Laminarisation of flow at low Reynolds number due to streamwise body force
}

\author{
S. $\mathrm{He}^{1, \dagger}, \mathrm{K} . \mathrm{He}^{1, \dagger}$ and M. Seddighi ${ }^{1,2}$ \\ ${ }^{1}$ Department of Mechanical Engineering, University of Sheffield, Sheffield S1 3JD, UK \\ ${ }^{2}$ Department of Maritime and Mechanical Engineering, Liverpool John Moores University, \\ Liverpool L3 3AF, UK
}

(Received 31 January 2016; revised 27 September 2016; accepted 7 October 2016; first published online 9 November 2016)

It is well established that when a turbulent flow is subjected to a non-uniform body force, the turbulence may be significantly suppressed in comparison with that of the flow of the same flow rate and hence the flow is said to be laminarised. This is the situation in buoyancy-aided mixed convection when severe heat transfer deterioration may occur. Here we report results of direct numerical simulations of flow with a linear or a step-change profile of body force. In contrast to the conventional view, we show that applying a body force to a turbulent flow while keeping the pressure force unchanged causes little changes to the key characteristics of the turbulence. In particular, the mixing characteristics of the turbulence represented by the turbulent viscosity remain largely unaffected. The so-called flow laminarisation due to a body force is in effect a reduction in the apparent Reynolds number of the flow, based on an apparent friction velocity associated with only the pressure force of the flow (i.e. excluding the contribution of the body force). The new understanding allows the level of the flow 'laminarisation' and when the full laminarisation occurs to be readily predicted. In terms of the near-wall turbulence structure, the numbers of ejections and sweeps are little influenced by the imposition of the body force, whereas the strength of each event may be enhanced if the coverage of the body force extends significantly away from the wall. The streamwise turbulent stress is usually increased in accordance with the observation of more and stronger elongated streaks, but the wall-normal and the circumferential turbulent stresses are largely unchanged.

Key words: buoyant boundary layers, pipe flow boundary layer, turbulence control

\section{Introduction}

Convective heat transfer, or simply convection, is commonplace in thermal energy systems. When the flow required for the convection to take place is provided by a pressure gradient such as that generated by a pump or a fan, it is referred to as forced

$\dagger$ Email address for correspondence: s.he@ sheffield.ac.uk

$\ddagger$ Present address: School of Chemistry and Chemical Engineering, South China University of Technology, Guangzhou 510640, China. 
convection. Alternatively, if the convection is due to a flow circulation driven by the buoyancy resulting from a non-uniform distribution of temperature in the fluid, it is referred to as natural convection. In many practical applications, the two forms of convection coexist; this combined forced and natural convection is also referred to as mixed convection.

Mixed convection in a vertical channel can be significantly stronger or weaker than the forced convection under the same flow rate. The latter is known as heat transfer deterioration and is a cause of concern in many applications, including for example the nuclear reactors under various normal and hypothetical fault conditions. The strengthening or reduction of heat transfer in mixed convection is largely due to the presence of the buoyancy, which influences heat transfer through the so-called structural (direct) or external (indirect) mechanisms (Petukhov, Polyakov \& Launder 1988). The former is due to the direct interactions between the fluctuating density (buoyancy) and the thermal field resulting in a production or destruction of turbulence. The latter is through an indirect means, that is, the buoyancy as a non-uniformly distributed body force causes the mean flow to be modified, leading to turbulence enhancement or suppression. In a vertical flow, the former is negligible; the latter is most influential (e.g. Jackson, Cotton \& Axcell 1989; Cotton \& Jackson 1990; Kim, He \& Jackson 2008). In a horizontal flow, both mechanisms might be significant.

Even though the detailed mixed convection behaviour in a vertical passage is very complex and difficult to predict, the general picture has been well established. In a heated downward flow (i.e. a buoyancy-opposed flow), turbulence is always enhanced as a result of the presence of buoyancy, and hence heat transfer is improved in comparison with forced convection. By contrast, in a heated upward flow (i.e. a buoyancy-aided flow), the production of turbulence is suppressed when the buoyancy is relatively weak. As the buoyancy force is increased (e.g. by increasing the heat flux and hence the wall temperature), turbulence continues to reduce causing the flow to partially relaminarise. When the buoyancy is sufficiently strong, the turbulence may be completely destroyed and the flow is fully laminarised. At this stage, the heat transfer process is minimised, being similar to that in a laminar flow. With a further increase in buoyancy force, turbulence starts to be produced again but mostly in the region away from the wall, which causes a recovery of heat transfer effectiveness. When the buoyancy is extremely strong and dominates the flow, the heat transfer process becomes a natural convection. At this stage, the Nusselt numbers for the upward and downward flows converge.

The present understanding of the mechanisms of the effect of buoyancy on heat transfer is explainable using the analysis due to Jackson \& Hall (1979). In a heated upward flow, the buoyancy force, which is strongest adjacent to the wall due to the large temperature gradient there, causes the flow in the viscous layer to accelerate strongly and the flow in the core to be retarded in relative terms. This causes the total shear and then the turbulent shear stress to reduce in the buffer layer, which in turn leads to a reduction in turbulence production. The opposite is true for a buoyancyopposed flow: the flow in the viscous sublayer is reduced resulting in a larger velocity gradient and a stronger turbulent shear stress, and hence an increase in turbulence production.

Extensive research has been carried out over many decades on mixed convection heat transfer with various fluids, generating a large body of experimental data. Most studies however are limited to heat transfer measurements, i.e. wall temperature and heat flux on the wall (e.g. Vilemas, Poskas \& Kaupas 1992; Celata et al. 1998; Liao \& Zhao 2002; Lee et al. 2008). An overview of earlier work can be found 
in Jackson et al. (1989) and more recently Jackson (2013). Alongside experimental investigations, various heat transfer correlations have been established based on dimensional arguments and/or mechanistic models (Petukhov et al. 1988; Jackson et al. 1989; Vilemas et al. 1992; Aicher \& Martin 1997). More recently, heat transfer to fluids at supercritical pressure has received significant attention due to a number of emerging applications, such as, advanced design of nuclear reactors, supercritical $\mathrm{CO}_{2}$ air-conditioning systems and carbon capture and storage (Jiang et al. 2006; Licht, Anderson \& Corradini 2009; Jackson 2013). The effect of buoyancy can be particularly strong and complex since the thermal properties vary strongly with temperature under such conditions. A review of recent studies can be found in Yoo (2013).

Computational fluid dynamics has been applied to reproduce mixed convection and one of the very first attempts was Abdelmeguid \& Spalding (1979). The standard $k-\epsilon$ model used was found to be able to reproduce the downward flow but not the upward flow. Efforts have been made to revise turbulence models to simulate buoyancy effect, using advanced wall functions (Craft et al. 2004), improved modelling of the buoyancy production using generalised gradient diffusion hypothesis (GGDH) or algebraic models (Cotton \& Jackson 1990), various turbulent shear stress models and turbulent heat transfer models (Kim et al. 2008). It has now been established that it is essential that the turbulence model has a so-called low Reynolds number feature, which emulates the effect of buoyancy on turbulence (Cotton \& Jackson 1990; Kim et al. 2008). Comparative studies (Mikielewicz et al. 2002; Kim et al. 2008; Keshmiri et al. 2012) have shown that although some models perform better than others, there are no models that can be applied generically in all flow conditions.

In contrast to the vast body of heat transfer measurements, there are very limited measurements of the flow and turbulence. One of the first detailed measurements of the velocity profiles was carried out by Steiner (1971) who studied the 'reverse transition' of a turbulent flow under the action of buoyancy forces. He demonstrated that the velocity profiles are flattened in flows with influences of weak and medium buoyancy forces when the flow is partially laminarised, and become M-shape when the buoyancy is very strong and turbulence and heat transfer effectiveness regain their strength. These were further confirmed by Carr, Connor \& Buhr (1973) and Polyakov \& Shindin (1988), both using air, and Kurganov \& Kaptilny (1992) using $\mathrm{CO}_{2}$ at a supercritical pressure. These later studies also provided measurements of the turbulent shear stress, which reduces first with the increase of buoyancy, reaching a minimum (close to zero) while the flow is said fully laminarised. With further increase of the buoyancy, negative turbulent shear stress is generated in the core of the flow which corresponds to the inner flank of the M-shaped velocity. Some positive turbulent shear stress is also produced close to the wall in connection with the near-wall layer (the outer legs of the M-shaped velocity profile). Simultaneous measurements of the axial and wall-normal turbulence were only made by Polyakov \& Shindin (1988) who discussed the anisotropy of the turbulence due to the influence of the buoyancy. They concluded that the axial turbulence is more strongly reduced than wall-normal turbulence causing the anisotropy of the turbulence to reduce. It is noted however that their data were limited to a strongly laminarised flow and some recovery flows. It is shown later that typically turbulence anisotropy is enhanced in a partially laminarised flow.

Studies of the flow and turbulence in a buoyancy-opposed flow are even rarer. Nakajima et al. (1980) made measurements of the streamwise velocity and temperature in a vertical plane channel with one heated and one cooled wall but only with 
moderate heat flux. Much later, Wang, Li \& Jackson (2004) made measurements of two components of turbulence flow in a vertical plane passage with a heated wall for a downward as well as an upward flow. In both studies, the results on the buoyancy-aided flow were consistent with those of previous studies in a tube, whereas the trends of the data for the buoyancy-opposed flow show contrasting features. The velocity in the viscous sublayer is retarded by the presence of buoyancy, leading to a stronger turbulence production which Wang et al. (2004) demonstrated with measurements of the turbulent normal and shear stresses.

More recently, direct numerical simulation (DNS) has been used to study combined forced and natural convection yielding detailed information on the flow and turbulence which are difficult, if not impossible, to obtain experimentally. Satake et al. (2000), You, Yoo \& Choi (2003) and Bae et al. (2006) performed DNS of air flows in a vertical tube, whereas Kasagi \& Nishimura (1997) considered a vertical plane channel with a heated and a cooled wall on either side using air-like properties based on the Boussinesq assumption. Bae, Yoo \& Choi (2005), Bae, Yoo \& McEligot (2008) performed DNS of $\mathrm{CO}_{2}$ at a supercritical pressure in a vertical circular and annular tube, respectively. Overall the DNS results agree well with the experiments where available, validating the numerical results as well as confirming the trends established from experiments. In addition, DNS also provide more detailed data, including the budget terms on turbulent stresses and turbulent heat fluxes. It has been demonstrated that in a partially laminarised flow, the wall-normal and spanwise turbulence components are significantly more strongly suppressed than the axial component, making the flow strongly anisotropic (Kasagi \& Nishimura 1997; Bae et al. 2006). This reflects the flow structures observed where elongated streaks are formed, which is typical of a laminarising flow due to flow acceleration, or, a favourable pressure gradient (Launder \& Jones 1969; Sreenivasan 1982; Lee \& Choi 2001; Piomelli \& Yuan 2013). Under most conditions, the contribution of the direct buoyancy production is insignificant and the influence of buoyancy on turbulence is largely via modifying the mean flow (Cotton \& Jackson 1990). In addition, DNS also shows that the flow and thermal fields can develop significant dissimilarity under some conditions invaliding the common turbulence modelling approach based on turbulent Prandtl number (Bae et al. 2005).

Even though the main motivation of the present study stems from mixed convection, it should be noted that there are many other types of body-force-influenced flows which are of significant interest, especially, in relation to flow control and drag reduction. These different types of body forces share many similarities. Kasagi \& Nishimura (1997) directly compared the combined forced and natural convection in a vertical channel with a channel flow subject to a uniform wall mass injection and suction on each of the walls by Sumitani \& Kasagi (1995), and a liquid metal flow in a channel subject to a Lorenz force due to a transverse magnetic field. They demonstrated strong similarities between the various flows in not only the mean velocity profiles and turbulence statistics but also the near-wall quasi-coherent structures characterised by elongated streaks. They attribute the similarities to having resulted from the near-wall force balance modified in a similar way by the additional body force or momentum transport. Crawford \& Karniadakis (1997) studied numerically a turbulent flow of low electric conductivity subject to streamwise electromagnetic forcing. They did not achieve drag reduction that they intended for, which was attributed to the increase of turbulent shear stress due to the increased vortex stretching term $\left(\overline{w^{\prime} \omega_{2}^{\prime}}\right)$ and the spanwise variation of the stress component $\left(\partial\left(\overline{u^{\prime} w^{\prime}}\right) / \partial x_{3}\right)$. Xu et al. (2007) performed DNS of a turbulent channel flow subject 
to a prescribed steady streamwise force in the form of a sine wave in the near-wall region. Sustained drag reduction was achieved when the force was limited to within 20 wall units, which was attributed to the effect of the forcing limiting the development of the Reynolds shear stress. When the amplitude of the forcing was high, the shear layer was found to break down generating its own turbulence, in turn increasing the wall friction. Lee \& Choi (2001) studied the effects of Lorenz force on near-wall turbulence structures using DNS by subjecting a channel flow to a magnetic field in all three directions. It was found that whereas the near-wall turbulence structures can be suppressed by the Lorenz force induced by magnetic field in all three directions, the Lorenz force induced by the wall-normal field which directly interact with the mean flow can do so most effectively. When the magnetic field is too strong, turbulence is increased leading to a drag increase. Turbulence structures are markedly elongated in the direction of the applied magnetic field when it is sufficiently strong, which was shown to be associated with a rapid decrease of the Joule dissipation in time.

\subsection{The scope of the paper}

It is clear from the brief review provided above that buoyancy may significantly suppress turbulence resulting in a severe heat transfer deterioration. This is attributed to the modification of the near-wall shear stress balance, resulting in a modified velocity profile. Under such conditions, the flow shows typical characteristics of a laminarising flow with elongated streaks and strengthened anisotropic turbulence. The thermal and flow fields are strongly coupled in mixed convection and need to be resolved simultaneously for any practical problems. However, from the point of view of fluid mechanics, the fundamental question is how the flow and turbulence respond to a buoyancy force of a certain distribution, which is the key process in mixed convection ultimately determining the effectiveness of heat transfer. Despite the vast amount of efforts devoted to mixed convection, the understanding of such physics is still limited. For example, the prediction of flow laminarisation and heat transfer deterioration is still largely through semi-empirical correlations based on mechanistic modelling and dimensional analysis with the support of experimental data. The purpose of the study reported herein is to develop new insights into flow laminarisation due to a non-uniform body force such as the buoyancy in mixed convection. We perform DNS of a turbulent flow in a pipe subjected to a prescribed non-uniform, streamwise, body force. Two typical distributions, i.e. a linear variation and a step change, are studied, the amplitude and coverage of which are independently and systematically varied. This systematic approach allows us to establish a holistic view of how a body force modifies the flow and turbulence, which could not have been developed through studying individual physical flows, which by definition are limited to certain conditions. We consider body-force aided flow, that is, the body force is in the same direction of the flow. We focus on partially and fully laminarised flows herein, while the recovery flow will be discussed in detail elsewhere. This study has led to the establishment of a new framework of analysis for the flow subjected to a body force, and has revealed some fundamental characteristics of laminarising flows for the first time. The new understanding provides explicit means to predicting how much the flow is modified or laminarised under the influence of a known body force.

The paper is structured as follows: the numerical methods and the design of the test cases are discussed in $\S 2$, which is followed by detailed discussion of the results in $\S 3$. The overall picture of the influence of systematically varying the body force on 
the velocity profile and turbulence is shown in $\S 3.1$ and the turbulence behaviour in a laminarising flow in the conventional view is described in $\S 3.2$. A new perspective and the underlying theory are detailed in $\$ 3.3$ together with supporting evidence. The friction factor and an FIK identity analysis (Fukagata, Iwamoto \& Kasagi 2002) are shown in $\$ 3.4$, followed by discussion on the fully laminarised flow process and its prediction in $\S 3.5$. The flow structures are visualised and quantified in $\S 3.6$ and finally the paper concludes with a summary in $\S 4$.

\section{Methodology}

The DNS code, CHAPSim, initially developed for a channel flow (Seddighi 2011; He \& Seddighi 2013) has been revised to solve the Navier-Stokes equations for a turbulent flow in a pipe in cylindrical coordinates. The governing equations read:

$$
\begin{gathered}
r \frac{\partial q_{z}}{\partial z}+\frac{\partial q_{r}}{\partial r}+\frac{1}{r} \frac{\partial q_{\theta}}{\partial \theta}=0 \\
\frac{\partial q_{z}}{\partial t}+\frac{\partial q_{z} q_{z}}{\partial z}+\frac{1}{r} \frac{\partial q_{z} q_{r}}{\partial r}+\frac{1}{r^{2}} \frac{\partial q_{z} q_{\theta}}{\partial \theta} \\
=-\frac{\partial p}{\partial z}-\frac{\partial P}{\partial z}+\frac{1}{R e}\left(\frac{\partial^{2} q_{z}}{\partial z^{2}}+\frac{1}{r} \frac{\partial}{\partial r} r \frac{\partial q_{z}}{\partial r}+\frac{1}{r^{2}} \frac{\partial^{2} q_{z}}{\partial \theta^{2}}\right)+f, \\
\frac{\partial q_{r}}{\partial t}+\frac{\partial q_{r} q_{z}}{\partial z}+\frac{\partial q_{z}\left(q_{r} / r\right)}{\partial r}+\frac{\partial\left(q_{\theta} q_{r}\right) / r^{2}}{\partial \theta}-\frac{q_{\theta}^{2}}{r^{2}} \\
=-r \frac{\partial p}{\partial r}+\frac{1}{R e}\left(\frac{\partial^{2} q_{r}}{\partial z^{2}}+\frac{\partial}{\partial r} r \frac{\partial\left(q_{r} / r\right)}{\partial r}+\frac{1}{r^{2}} \frac{\partial^{2} q_{r}}{\partial \theta^{2}}-\frac{q_{r}}{r^{2}}-\frac{2}{r^{2}} \frac{\partial q_{\theta}}{\partial \theta}\right), \\
\frac{\partial q_{\theta}}{\partial t}+\frac{\partial q_{\theta} q_{z}}{\partial z}+\frac{\partial q_{r}\left(q_{\theta} / r\right)}{\partial r}+\frac{1}{r^{2}} \frac{\partial\left(q_{\theta} q_{\theta}\right)}{\partial \theta}+\frac{q_{\theta} q_{r}}{r^{2}} \\
=-\frac{\partial p}{\partial \theta}+\frac{1}{R e}\left(\frac{\partial^{2} q_{\theta}}{\partial z^{2}}+\frac{\partial}{\partial r} r \frac{\partial\left(q_{\theta} / r\right)}{\partial r}+\frac{1}{r^{2}} \frac{\partial^{2} q_{\theta}}{\partial \theta^{2}}-\frac{q_{\theta}}{r^{2}}+\frac{2}{r^{2}} \frac{\partial q_{r}}{\partial \theta}\right) .
\end{gathered}
$$

The $z, r, \theta$ are respectively the streamwise, radial and azimuthal coordinates and $q_{z}=u_{z}, q_{r}=r u_{r}, q_{\theta}=r u_{\theta}$ are three fluxes introduced to circumvent the singularity on the axis of the pipe (Orlandi 2000). In addition, the wall-normal distance, $y(=1-r)$, is also sometimes used. The equations are non-dimensionalised using the pipe radius $R^{*}$, the density $\rho^{*}$ and the centre velocity of the laminar Poiseuille flow $U_{P}^{*}$ (which is 2 times the bulk velocity $U^{*}$ ), where the superscript ' $*$ ' indicates that the quantity is not non-dimensionalised. Unless otherwise stated, any quantities shown without a superscript are always normalised by these quantities. The Reynolds number $R e=\rho^{*} U_{P}^{*} R^{*} / \mu^{*}$. The prescribed body force is normalised in the following form: $f=f^{*} /\left(\rho^{*} U_{P}^{* 2} / R^{*}\right)$. The pressure is split into a domain average $(P)$ and fluctuations around it $(p)$ and the former is a function of $z$ only. The spatial derivatives are discretised using a second-order central finite difference method. An explicit low-storage, third-order Runge-Kutta scheme is used for the temporal discretisation of the nonlinear terms and a second-order implicit Crank-Nicholson scheme is used for other terms. These are combined with the fractional-step method to enforce the continuity constraint (Orlandi 2000). Periodic boundary conditions are applied in the axial and azimuthal directions, and a no-slip boundary condition is imposed at 


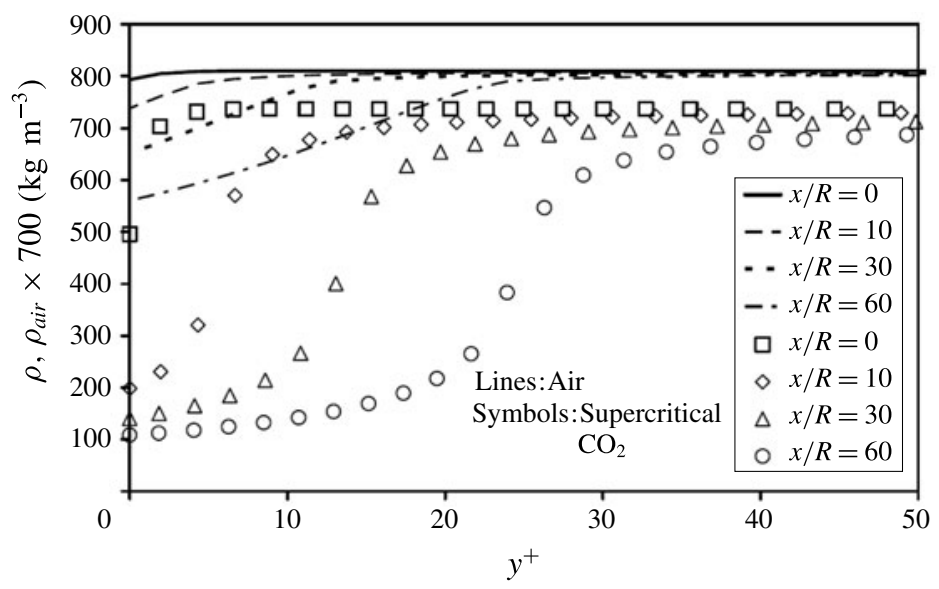

FIGURE 1. Examples of distributions of density in mixed convection of a flow of air and $\mathrm{CO}_{2}$ at supercritical pressure. ' $x / R$ ' is the distance from the start of the heated length of the pipe over radius. (Reproduced from He, Kim \& Bae (2008) with permission.)

the wall. The message passing interface (MPI) is used to parallelise the code. More detailed descriptions can be found in Seddighi (2011) and the validation of the code for pipe flows is given in He, Seddighi \& He (2016).

In order to determine the body force to be used, we inspect the buoyancy force distribution in some typical mixed convection flows. Figure 1 shows the buoyancy force distributions in the first $60 R^{*}$ of an upward flow of air and supercritical $\mathrm{CO}_{2}$ in a heated tube (He et al. 2008). Since the buoyancy results from the variation of the temperature of the fluid, it is strongest at the wall, reducing away from the wall over a region that is largely corresponding to the thermal boundary layer. Figure 1 shows that the buoyancy varies approximately linearly in the air flow, whereas a near step change is observed in the flow of supercritical $\mathrm{CO}_{2}$. The latter occurs at the location where the temperature of the fluid is around the pseudo-critical point across which a large change in density occurs. In both cases, the significant changes in buoyancy force are within a region where $y^{+}<50$. Keeping these in mind, we have chosen a linear and a step distribution defined in the following forms to study:

Linear variation:

$$
f= \begin{cases}a\left(r-r_{0}\right) /\left(1-r_{0}\right) & r \geqslant r_{0} \\ 0, & r<r_{0}\end{cases}
$$

and step variation:

$$
f= \begin{cases}a & r \geqslant r_{0} \\ 0, & r<r_{0}\end{cases}
$$

The body force is therefore steady in time, and uniform in the streamwise and circumferential directions. The cross-sectional average is $F=\int_{0}^{1} 2 r f \mathrm{~d} r$. Hence the body force is characterised by its coverage (represented by $r_{0}$ ), the amplitude $a$ and the 'strength' of the body force represented by $F$. Four groups of test flows have been carried out, the body-force distributions of which are shown in figure 2. Groups A and B employ a linearly varying body force to study the coverage and amplitude effects respectively, whereas groups $\mathrm{C}$ and $\mathrm{D}$ employ a step-change body force again studying the coverage and amplitude effects respectively. Within each group, the 


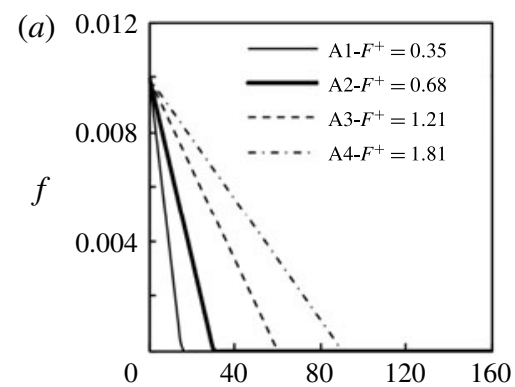

(b)
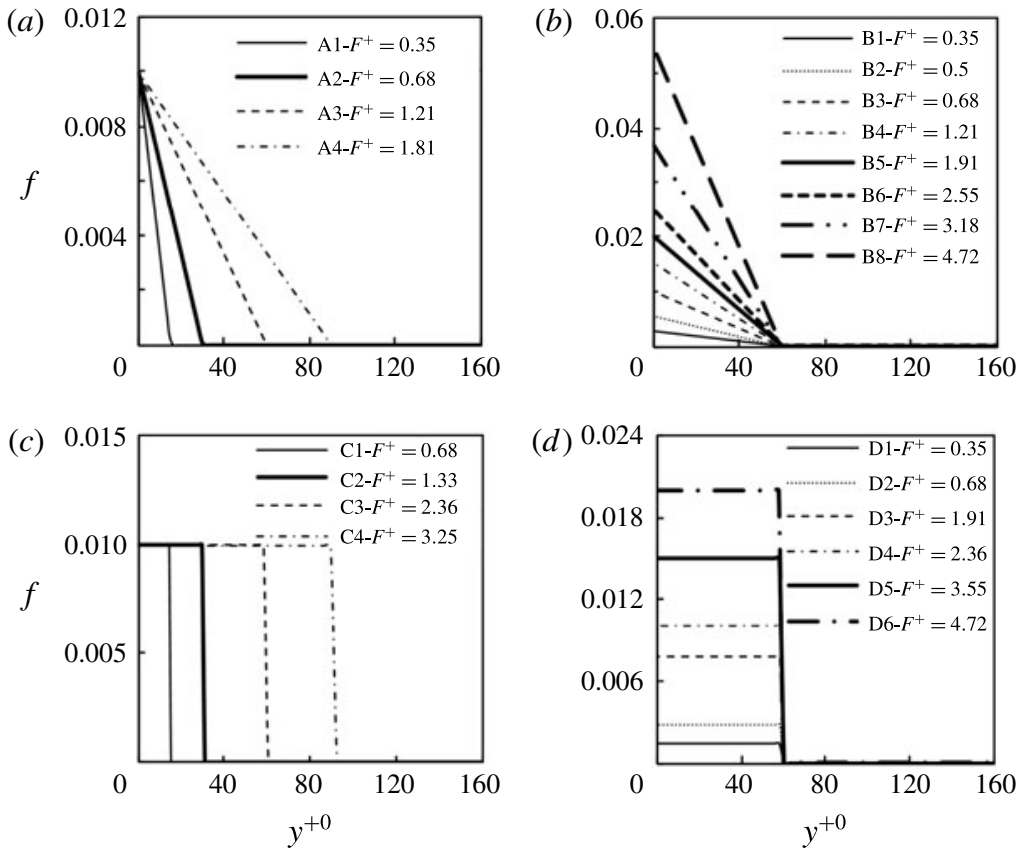

FIGURE 2. Body-force distributions $\left(y^{+0}=y^{*} u_{\tau 0}^{*} / \nu^{*}, F^{+}=F^{*} /\left(2 \tau_{w}^{*} R^{*}\right)\right.$ where $u_{\tau 0}^{*}$ and $\tau_{w 0}^{*}$ are the friction velocity and the wall shear stress of the base flow, $R e_{\tau}=180$, respectively).

$\begin{array}{lcccccc}\text { Case } & \Delta_{z}^{+} & \Delta(r \theta)_{\max }^{+} & \Delta r_{\max }^{+} & \Delta r_{\min }^{+} & R e_{\tau} & R e \\ \text { Base } & 6 & 4.7 & 3.6 & 0.14 & 180 & 5300 \\ \text { A1-A4 } & 6.2-6.4 & 4.9-5 & 3.7-3.9 & 0.14-0.15 & 187-193 & 5300 \\ \text { B1-B8 } & 5.3-9.2 & 4.1-7.2 & 3.2-5.5 & 0.12-0.21 & 159-276 & 5300 \\ \text { C1-C4 } & 6.3-6.7 & 5-5.2 & 3.8-4 & 0.15-0.16 & 191-200 & 5300 \\ \text { D1-D6 } & 4.8-8.4 & 3.8-6.5 & 2.9-5 & 0.11-0.2 & 144-231 & 5300 \\ \text { B21 } & 7.3 & 5.7 & 4.4 & 0.17 & 220 & 6819\end{array}$

TABLE 1. Mesh sizes of the test cases $\left(R e_{\tau}=u_{\tau}^{*} R^{*} / \nu^{*}, R e=U_{P}^{*} R^{*} / \nu^{*}\right.$, where $U_{P}^{*}$ is the centre velocity of the laminar Poiseuille flow).

strengths of the body forces are chosen so that typical flow patterns encountered in mixed convection flows, i.e. partially and fully laminarised and recovery flows, are all represented (see $\S 3.1$ ).

The simulations are conducted for a pipe of a length of $20 R^{*}$ with a mesh of $600 \times 96 \times 240$ in streamwise $(z) \times \operatorname{radial}(r) \times$ circumferential $(\theta)$ directions, respectively. The pipe length chosen here is slightly longer than those commonly used in the literature (e.g. Kim, Moin \& Moser 1987; Wu \& Moin 2008) considering the low Reynolds number flows concerned. It is also interesting to note that Tsukahara et al. (2014) showed that a box of a length $25.6 \delta^{*}$ can sustain turbulence for a channel flow at $R e_{\tau} \approx 64$. The range of mesh resolution of each of the test groups is shown in table 1. The worst case is B8, the resolution of which is however still within the range of common recommendations. A direct validation of a mesh of similar resolution using CHAPSim against benchmark data from literature was reported in a 


$\begin{array}{lcccc}\text { Case } & F^{+} & U_{b f} & R e_{\tau p} & \text { EPG reference flow } \\ \text { A1 } & 0.35 & 0.057 & 160 & R e_{\tau}=157 \\ \text { A2 } & 0.68 & 0.209 & 110 & R e_{\tau}=112 \\ \text { B1 } & 0.35 & 0.142 & 136 & R e_{\tau}=135 \\ \text { B2 } & 0.50 & 0.232 & 105 & R e_{\tau}=112 \\ \text { C1 } & 0.68 & 0.166 & 125 & R e_{\tau}=129 \\ \text { D1 } & 0.35 & 0.167 & 138 & R e_{\tau}=135 \\ \text { B21 } & 0.50 & 0.169 & 178 & R e_{\tau}=180\end{array}$

TABLE 2. Additional flow parameters for the laminarising flow cases. $F^{+}=F^{*} /\left(2 \tau_{w 0}^{*} R^{*}\right)$ is the normalised cross-section-averaged body force and $\tau_{w 0}$ is the wall shear stress of the base flow, $R e_{\tau}=180 ; U_{b f}$ is the bulk velocity of the body-force induced perturbation flow and $R e_{\tau p}$ is the apparent Reynolds number. The last column shows 'standard' flows simulated (i.e. those without any non-uniform body force) which have values of $R e_{\tau}$ closest to those of $R e_{\tau p}$ of the corresponding flow and hence serve as their EPG reference flows for the respective cases.

previous study (He et al. 2016). For any test case, the body force $(f)$ is implemented as fixed values according to figure 2, and the pressure gradient will adjust itself so as to maintain the Reynolds number $R e$ (and hence the mass flow rate) constant at a prescribed value. For all cases except B21, $R e=5300$. Case B21 is conducted with a fixed pressure gradient that is the same as that of the base flow $\left(R e_{\tau}=180\right)$ and a body force that is the same as that of case B2. The statistical results are obtained via ensemble averaging in the two periodic directions as well as over at least 30 independent flow fields after the flow is fully developed in time. Results for cases shown in table 2 are based on 100 flow fields.

\section{Results and discussion}

\subsection{The general picture}

Figures 3 and 4 show the velocity profiles of all the test cases in linear global coordinates and semi-logarithmic wall coordinates, respectively. With the increase of the body force $(F)$, through either increasing the coverage or the amplitude, the general trends of the variations of the velocity profiles in each of the groups are similar to each other. We focus on group B to elucidate such trends but comment on the differences between the groups as appropriate. With a relatively low body force (cases B1 to B3), the velocity profile is distorted only slightly, becoming flattened in the core of the flow. The centreline velocity becomes lower than that of the reference case, and the velocity around say $y=0.3$ becomes higher. The velocity gradient near the wall is always lower than that of the reference flow, and decreasing with the increase of the body force. This is however not always the case in other groups. For example, the velocity gradient adjacent to the wall in A1 and C1 is higher than in the reference flow, and hence under such conditions the friction factor is increased in comparison with the reference flow even though the flow is partially laminarised (see later). Further increasing the body force, the velocity profiles suddenly switch to an M-shape, which is typical of mixed convection with a strong influence of buoyancy (e.g. Steiner 1971; Carr et al. 1973; You et al. 2003). With the increase of the body force, the velocity in the centre of the pipe reduces and the velocity gradient adjacent to the wall increases, and as a result, the friction factor also increases. The variation of the velocity profiles with increase of the body force follows a similar trend in 


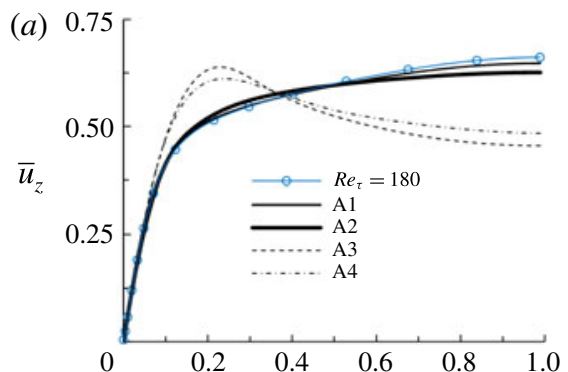

(b) 0.75
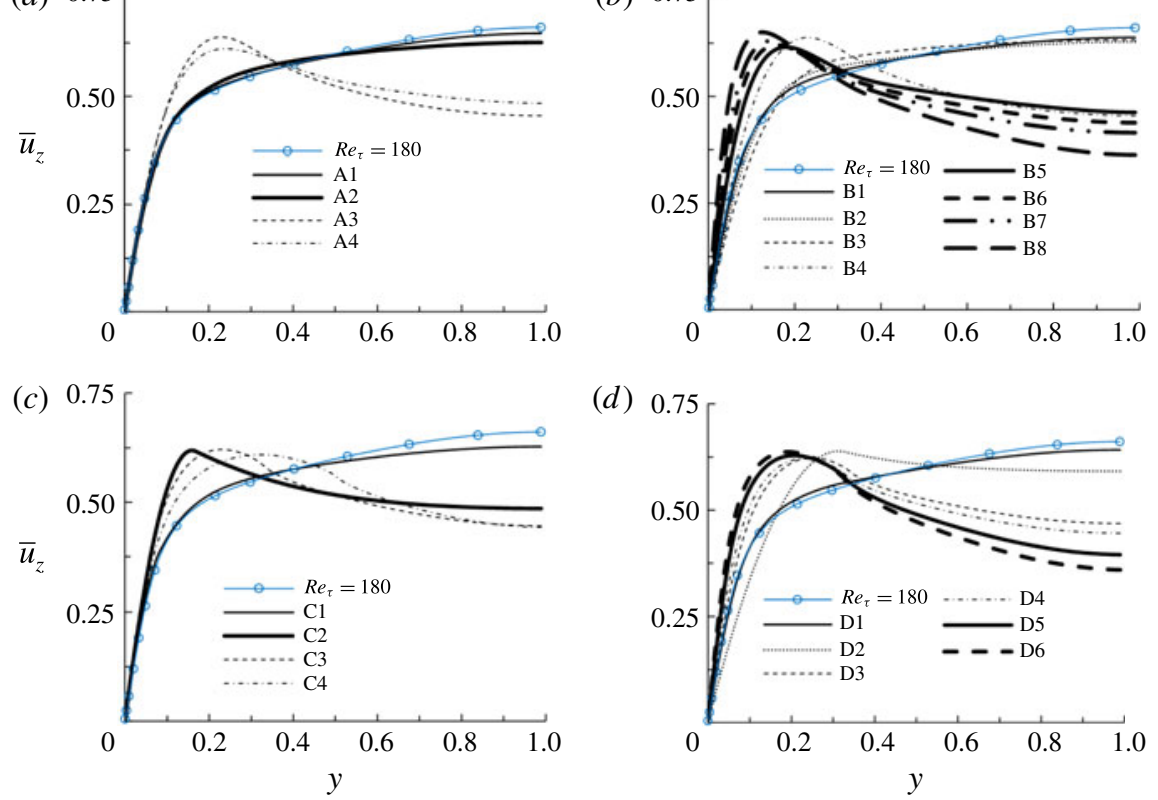

FIGURE 3. (Colour online) Mean velocity profiles of all test cases.

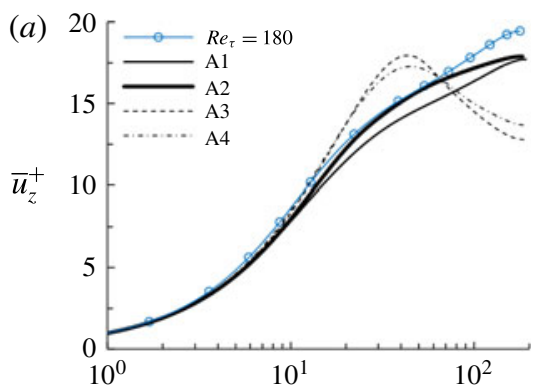

(b)
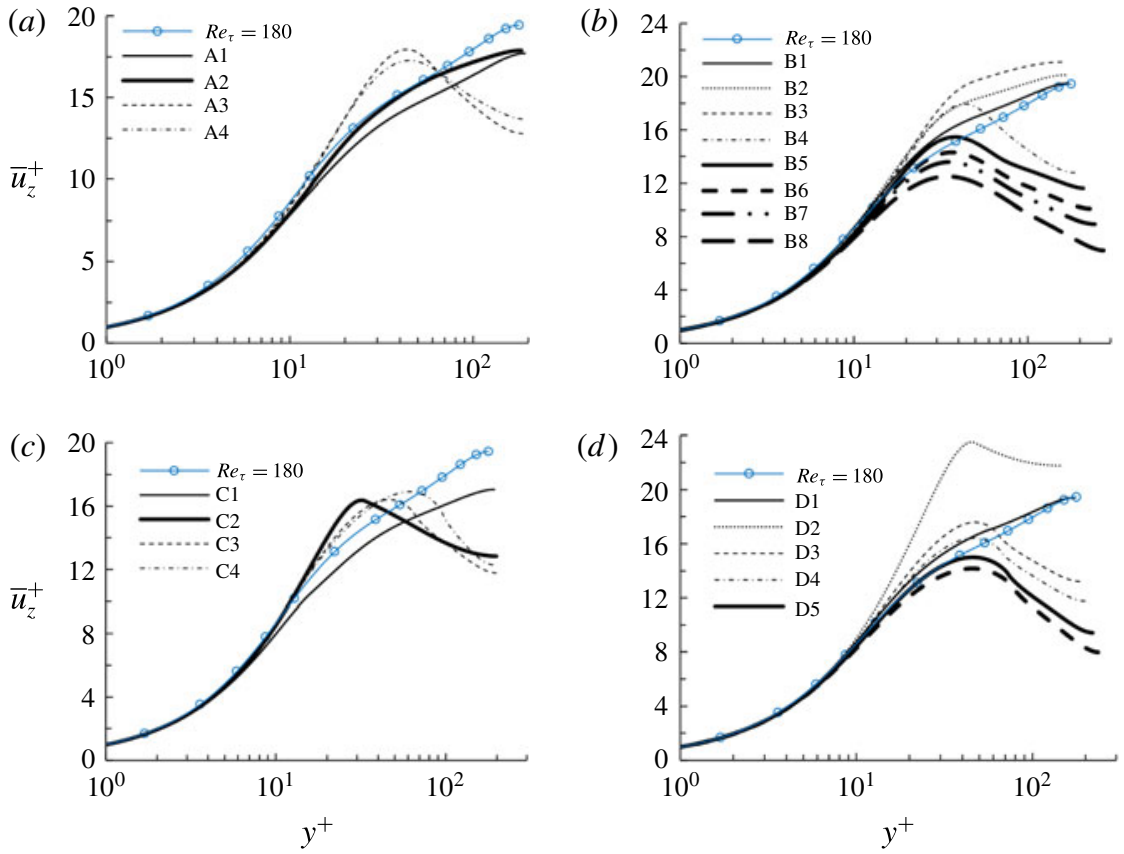

FIGURE 4. (Colour online) Mean velocity profiles of all test cases in wall coordinates.

group D, but in groups $\mathrm{A}$ and $\mathrm{C}$, the velocity gradient near the wall remains largely the same in the various test cases. That is, the overall friction is insensitive to the increase of the imposed body force under such conditions. 

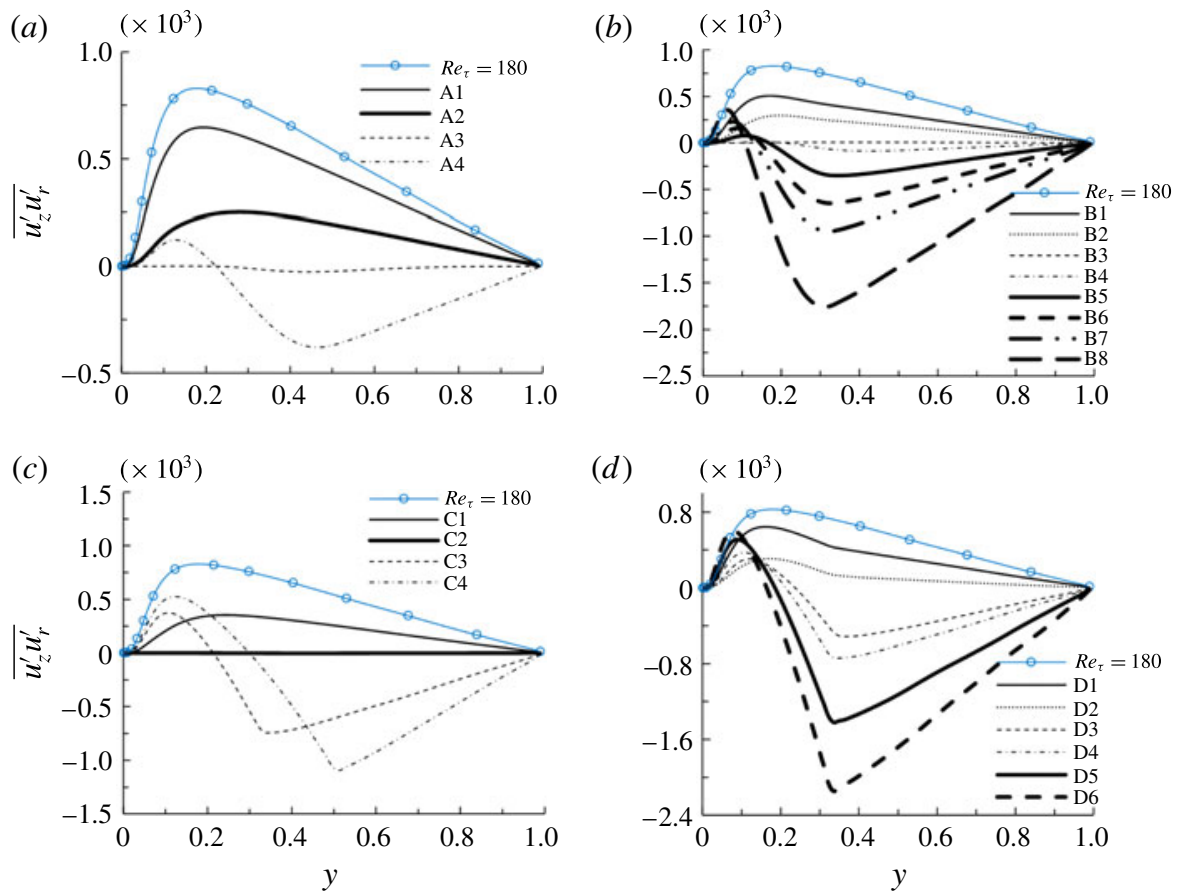

Figure 5. (Colour online) Distribution of turbulent shear stress in all test cases.

Figure 4 shows how far the velocity profiles can still be described by the generic logarithmic distribution. For the weaker body-force-influenced cases (B1 to B3), the velocity is upshifted with increase of the body force, showing increasingly stronger laminarisation. There is always a region of logarithmic distribution (linear variation in the semi-log plot) in each case, but the gradient becomes steeper with increase of the body force. In consistent with the observation in figure 3, the profiles of cases $\mathrm{A} 1$ and $\mathrm{C} 1$ are actually shifted downwards as a result of the increase of the friction velocity. The plots of the M-shaped velocity profiles have little meaning in this style of presentation, except that it does show that for most cases, the profiles follow the 'standard' curve up to $y^{+}=15$, and then either curve up or down first before going downwards in the core of the flow.

To illustrate the effect of the body force on turbulence, the turbulent shear stress $\left(\overline{u_{z}^{\prime} u_{r}^{\prime}}\right)$ and kinetic energy $(k)$ are shown in figures 5 and 6 , respectively. When the body force is relatively weak, say, B1 to B3, both turbulent shear stress and kinetic energy reduce progressively with increase of the body force. In fact, the flow is effectively completely laminarised in B3 (and A3 and C2), where both $\overline{u_{z}^{\prime} u_{r}^{\prime}}$ and $k$ are negligibly small. With a further increase of the body force, negative shear stresses are generated in the core of the flow, and the magnitude of which increases with the increase of the body force. At the same time, positive shear stress also starts to appear in the wall region. The peak value increases with increase of the body force, and its location moves closer to the wall. The production of negative turbulent shear stress stems from the velocity gradient of the M-shaped profile in the core region, which has been observed by various researchers previously (e.g. Polyakov \& Shindin 1988; Bae et al. 2005). Along with the increase of $\overline{u_{z}^{\prime} u_{r}^{\prime}}$, turbulent kinetic energy also increases with increase of the body force. There are two peaks in the distribution of $k$, one is clearly 

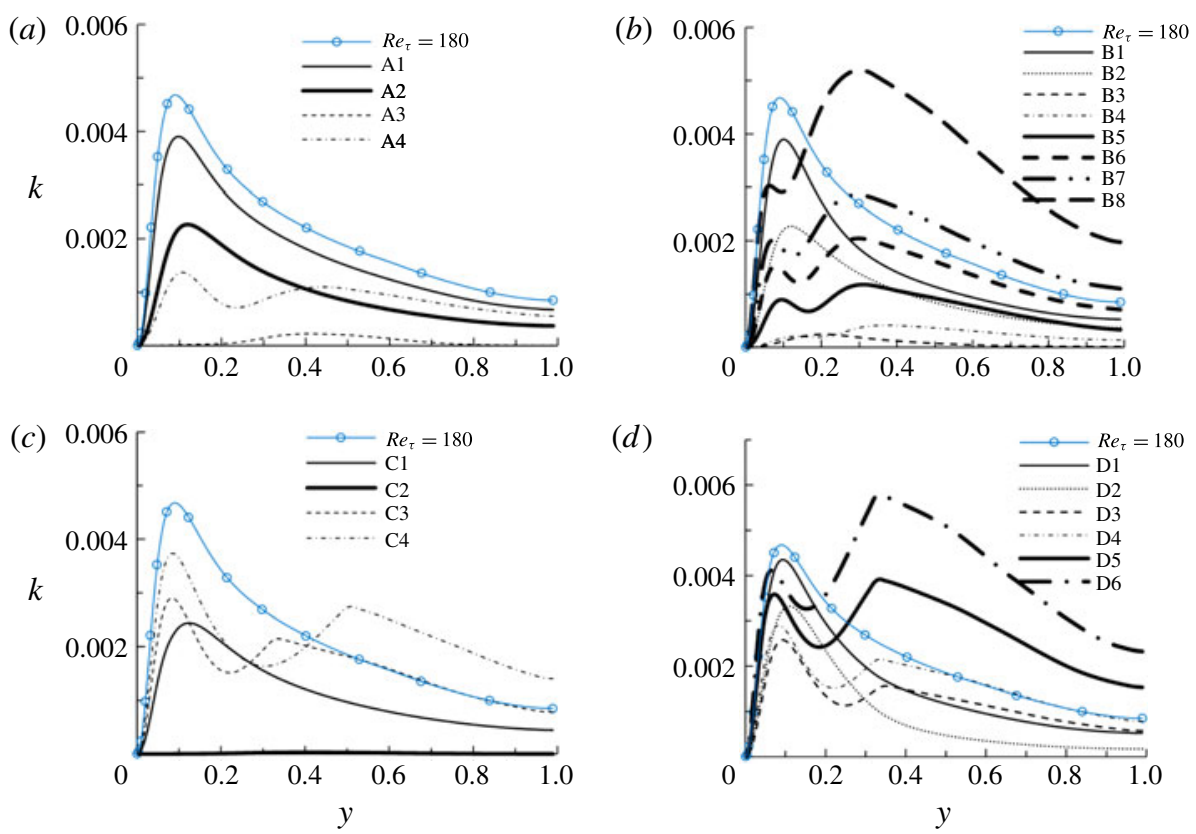

FIGURE 6. (Colour online) Distribution of turbulent kinetic energy in all test cases.

associated with the wall layer (the outer legs of the M) and the other is associated with the inner flank of the M-profile (the core of the flow), linked to the negative turbulent shear stress.

According to the effect on turbulence, the body-force-aided flows can be grouped into three categories (regimes), that is, partially laminarised (or, laminarising), fully laminarised or recovery flows. In a partially laminarised flow, turbulent kinetic energy and shear stress are both reduced in magnitude, and the reduction becomes stronger with the increase of the body force. The velocity profile is flattened in the core of the flow, but is overall only slightly distorted from that of the reference flow. In the recovery regime, the velocity takes an M-shape, where the inner flank of the M profile provides the reason for the generation of turbulence in the core of the flow. The stronger the body force, the stronger the turbulence becomes. In between the above two scenarios, turbulence completely or largely disappears. In such a fully laminarised flow, the velocity profile can take either of the above shapes.

The above results show a comprehensive picture of how the flow and turbulence vary with a systematically varied body force imposed through varying the amplitude or the coverage. It is interesting to note that a step-change body force can achieve a similar result as a linearly varying body force, and that increasing the amplitude and the coverage can also achieve similar results. The present results with systematically varied body force distributions of two distinct profiles provide an opportunity to gain new insights into how the body force modifies the flow and turbulence, which is discussed later in this paper. An inevitable limitation of previous studies on mixed convection is that the range of conditions covered is always limited. A complete picture such as that shown above has not previously been observed in a single study. The measurements of Shehata \& McEligot (1998) and DNS of Satake et al. (2000) and Bae et al. (2006) were limited to flows in the laminarising regime. The measurements of Carr et al. (1973) and the DNS of You et al. (2003) observed 

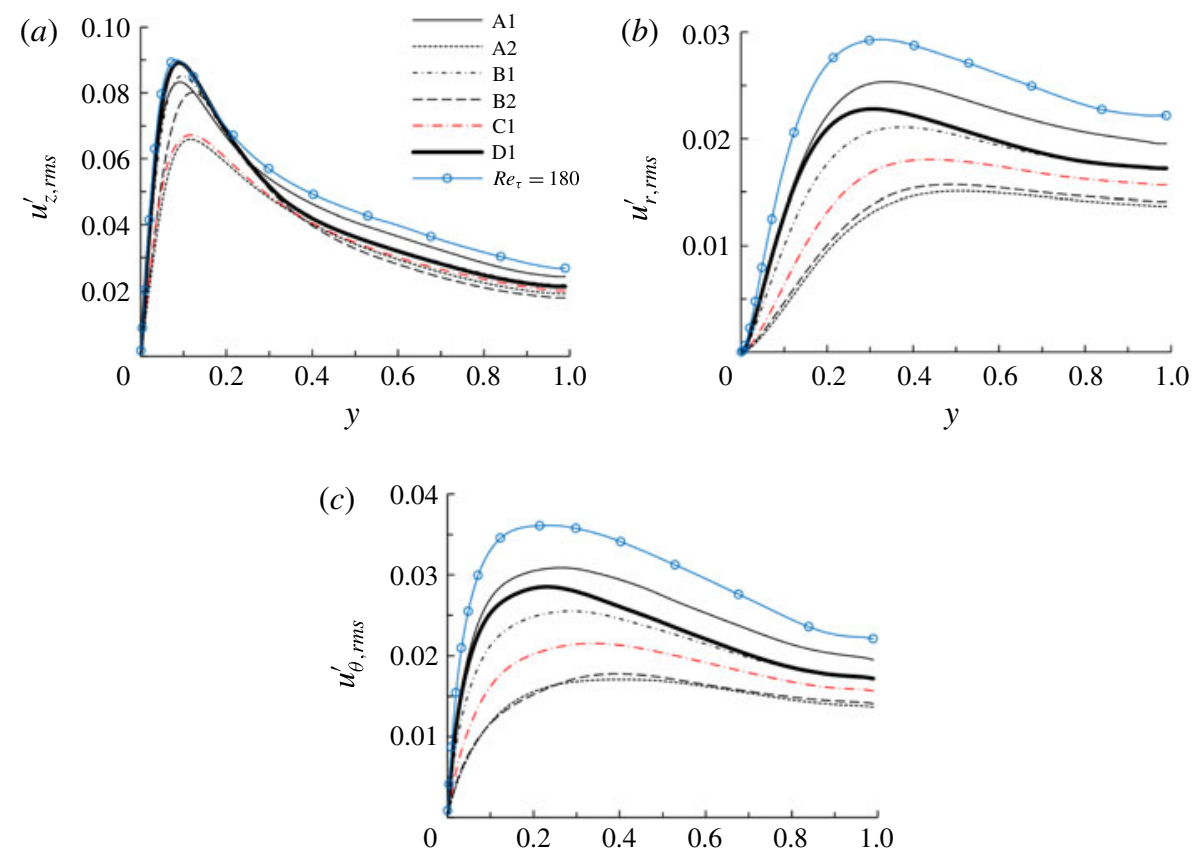

FIGURE 7. (Colour online) Distribution of the root-mean-square of the turbulent velocities.

both types of the velocity profiles, but their strongest buoyancy flows saw only very small recovery of turbulence. In Bae et al.'s (2005) DNS of mixed convection of $\mathrm{CO}_{2}$ at supercritical pressure, the flow undergoes the process of first relaminarisation and then strong recovery along the length of the heated pipe, illustrating the various stages of buoyancy effect on the mean flow and turbulence as observed herein. Their study was however for only one flow condition (and a particular distribution of the body force). Polyakov \& Shindin (1988) carried out measurements of velocity and turbulence for a wide range of buoyancy values. A limitation of that work is however that the partially laminarising flow that they chose to study was actually close to be fully laminarised, and as a result, they observed the turbulence becoming more isotropic as the flow is laminarised, which is in contrast to the observations of all other studies.

Hereafter, we focus on the partially laminarised flow, and to a lesser extent the fully laminarised flow. The recovery flow will be further studied elsewhere.

\subsection{Turbulence in relaminarising flow - the conventional viewpoint}

Figure 7 shows the three components of the turbulent normal stresses normalised using the centreline velocity of the laminar Poiseuille flow. The turbulent stresses reduce in all the cases though some of the reductions are stronger than others. More significantly, the reductions in the wall-normal and circumferential components are always much stronger than that in the streamwise component, which is consistent with the observation of laminarising flow in mixed convection (Kasagi \& Nishimura 1997; Bae et al. 2006). The cases with the strongest reductions in $u_{r}^{\prime}$ and $u_{\theta}^{\prime}$ are cases A2 and B2, where they reduce to approximately a half of that of the reference case. By contrast, the reduction in $u_{z}^{\prime}$ is only approximately $30 \%$ and $10 \%$ in cases $\mathrm{A} 2$ and $\mathrm{B} 2$ respectively. For case D1 the peak values of the $u_{r}^{\prime}$ and $u_{\theta}^{\prime}$ reduce by 

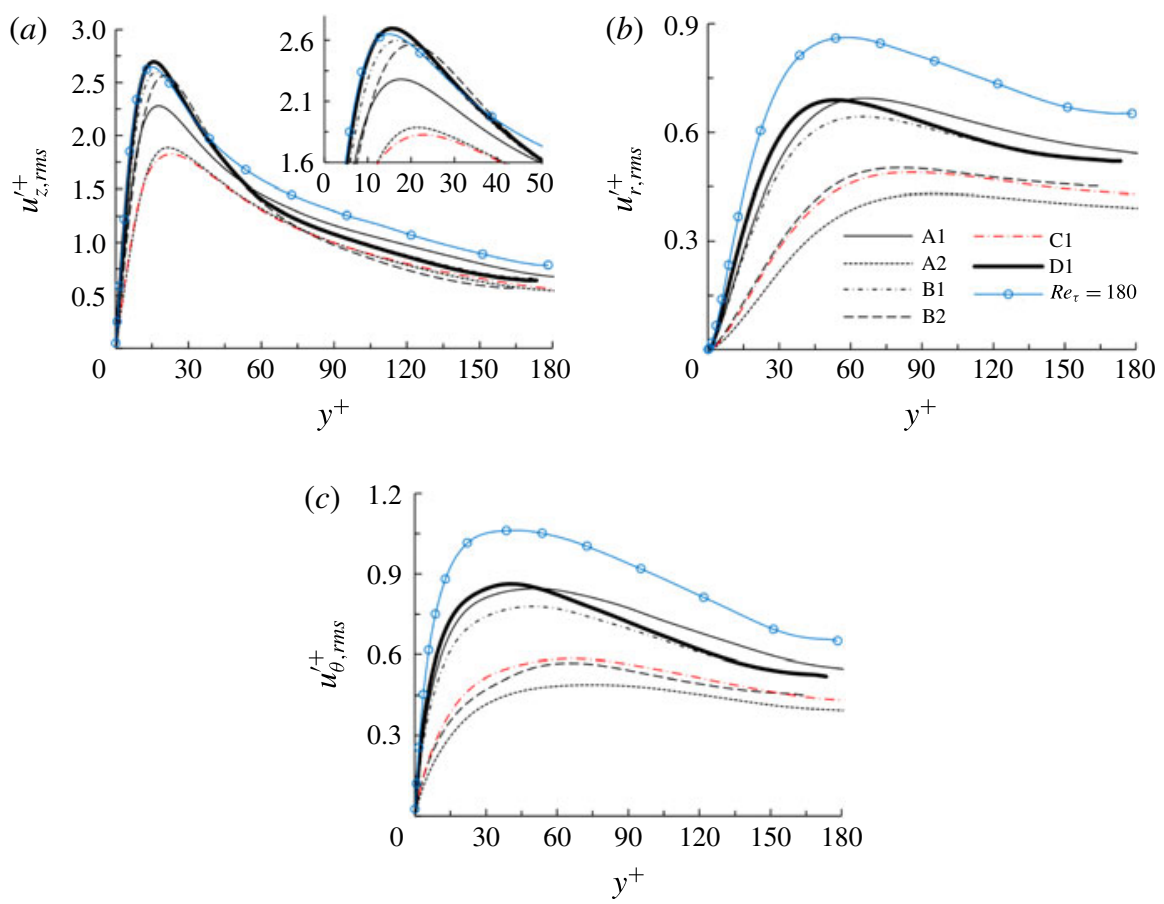

FIGURE 8. (Colour online) Distribution of the root-mean-square of the turbulent velocities in wall units.

about $30 \%$, but the peak value of the $u_{z}^{\prime}$ remains more or less the same as that of the reference case near the wall, even though the values are clearly reduced in the core. As a result of such unequal reduction in the three components, the turbulence becomes strongly anisotropic which is known to be a characteristic of a laminarising flow (e.g. Piomelli, Balaras \& Pascarelli 2000; Lee \& Choi 2001).

In the above, the discussion of turbulence reduction is based on absolute values since the normalisation uses the same constant $\left(U_{P}^{*}\right)$ in all the cases. In figure 8 , the same results are presented in wall coordinates, which enable us to study how far the turbulence in a laminarising flow deviates from the 'generic' distribution of an equilibrium turbulent flow. Again the turbulence quantities show various levels of reductions in all the cases studied. The reduction is stronger in $u_{r, r m s}^{\prime+}$ and $u_{\theta, r m s}^{\prime+}$ and less so in $u_{z, r m s}^{\prime+}$. However, the percentage of reduction has changed more significantly in some cases than in others, leading to a change of the order of the severity of laminarisation of the flows. For example, the peak value of $u_{z, r m s}^{\prime}$ is lower in B2 than in $\mathrm{A} 1$ but the opposite is true in $u_{z, r m s}^{\prime+}$ presentation. Overall, the turbulence reduction can be stronger or less strong in the absolute terms or in wall units depending on the flow conditions, which makes the prediction more difficult. Additionally, the location of the peak $u_{z, r m s}^{\prime+}$ has shifted away from the wall in the laminarising flows which has been observed in mixed convection under similar flow conditions (Carr et al. 1973; Bae et al. 2005).

\subsection{A new perspective on the relaminarisation of flow}

Ordinarily, comparisons of flows under various conditions are based on equal Reynolds numbers, which is the basis of the discussion presented above. For a 
given flow rate, the turbulence in a flow subjected to a non-uniform body force may be significantly lower than that in the 'undisturbed' flow of the same Reynolds number where the flow is driven by only a pressure force (which is uniform on any cross-section of the pipe). Under such a condition, the turbulence intensity, $k^{* 1 / 2} / U^{*}$, where $k^{*}$ is turbulent kinetic energy and $U^{*}$ the bulk velocity, is lower in the body-force-influenced flow. We therefore refer to such a flow to be (partially) laminarised, examples of which include, mixed convection in a vertical tube (Steiner 1971; Polyakov \& Shindin 1988), accelerating flows (Escudier et al. 1998; Piomelli et al. 2000; Greenblatt \& Moss 2004) and flows subject to certain control mechanisms (Lee \& Choi 2001; Xu et al. 2007), as discussed in the introduction.

Herein, we establish a new interpretation of the flow phenomena, which is in strong contrast to the above conventional view. We show that the body-force-influenced flow behaves as a 'standard' undisturbed turbulent flow but at a lower apparent Reynolds number; and this apparent Reynolds number is directly associated with the pressure gradient of the body-force-influenced flow. The understanding is supported by the following hypothesis which is established from the observations of the results reported herein: the addition of a body force to a flow that is initially driven by a pressure gradient only does not change the turbulent mixing characteristics of the initial flow and, in particular, the turbulent viscosity remains largely unchanged.

In the remaining part of the paper, we provide evidence supporting the above statement, and apply the hypothesis to characterising the laminarising flow under the influence of a body force. Furthermore, we demonstrate the simplicity of flow analysis in the new framework, and reveal new fundamental characteristics of laminarising flows that are difficult to identify in the classical viewpoint.

Let us first illustrate the implications of the hypothesis based on the Reynoldsaveraged analysis. The governing equation for a statistically steady flow of an incompressible fluid in a pipe, sufficiently away from the entrance is written as:

$$
0=-\frac{\mathrm{d} P}{\mathrm{~d} x}+\frac{1}{r} \frac{\partial}{\partial r}\left[r\left(\frac{1}{\operatorname{Re}} \frac{\partial \bar{u}_{z}}{\partial r}-\overline{u_{z}^{\prime} u_{r}^{\prime}}\right)\right]+f,
$$

where the pressure gradient $-\mathrm{d} P / \mathrm{d} x$ is the main driving force being constant in time and space; and $f$ is an additional body force varying along the radius, but uniform axially and circumferentially, and constant in time. This body-force-influenced flow can be compared with two 'standard' undisturbed flows without any non-uniform body forces: a flow with the same flow rate or a flow with the same pressure gradient. Herein we refer to them as the equal flow rate (EFR) reference flow and the equal pressure gradient (EPG) reference flow, respectively. All cases except B21 are performed at the same flow rate/Reynolds number, $R e=5300$, and hence share the same EFR reference flow, which is simply referred to as the base flow when there is no ambiguity, see table 1 . In the literature, comparisons between flows are commonly made based on equal flow rate as noted earlier. Below we compare the body-force-influenced flows with their EPG reference flow; the latter is denoted with a subscript ' $p$ ',

$$
0=-\left(\frac{\mathrm{d} P}{\mathrm{~d} x}\right)_{p}+\frac{1}{r} \frac{\partial}{\partial r}\left[r\left(\frac{1}{\operatorname{Re}} \frac{\partial\left(\bar{u}_{z}\right)_{p}}{\partial r}-\left(\overline{u_{z}^{\prime} u_{r}^{\prime}}\right)_{p}\right)\right]
$$


where $-(\mathrm{d} P / \mathrm{d} x)_{p}=-(\mathrm{d} P / \mathrm{d} x)$, that is, the pressure gradient of this (EPG) reference flow is the same as that of the body-force-influenced flow to be compared with. Subtracting (3.2) from (3.1),

$$
0=\frac{1}{r} \frac{\partial}{\partial r}\left[r\left(\frac{1}{\operatorname{Re}} \frac{\partial\left(\bar{u}_{z}\right)_{b}}{\partial r}-\left(\overline{u_{z}^{\prime} u_{r}^{\prime}}\right)_{b}\right)\right]+f
$$

where

$$
\left(\bar{u}_{z}\right)_{b}=\bar{u}_{z}-\left(\bar{u}_{z}\right)_{p} \quad \text { and } \quad\left(\overline{u_{z}^{\prime} u_{r}^{\prime}}\right)_{b}=\overline{u_{z}^{\prime} u_{r}^{\prime}}-\left(\overline{u_{z}^{\prime} u_{r}^{\prime}}\right)_{p}
$$

which represents a perturbation flow caused by imposing a non-uniform body force to a flow while maintaining the pressure gradient constant. This flow is denoted with a subscript ' $b$ '. Introducing the eddy viscosity concept, i.e. $\overline{u_{z}^{\prime} u_{r}^{\prime}}=-\left(v_{t} / \operatorname{Re}\right)\left(\partial \bar{u}_{z} / \partial r\right)$, where $v_{t}=v_{t}^{*} / \nu^{*}$ and $v_{t}^{*}$ is the turbulent viscosity, the above equation can be written as:

$$
0=\frac{1}{r} \frac{\partial}{\partial r}\left[\frac{r}{R e}\left(\frac{\partial\left(\bar{u}_{z}\right)_{b}}{\partial r}+v_{t} \frac{\partial \bar{u}_{z}}{\partial r}-v_{t 1} \frac{\partial\left(\bar{u}_{z}\right)_{p}}{\partial r}\right)\right]+f .
$$

Now applying the hypothesis introduced above, i.e. the turbulent viscosity remains uninfluenced by the imposition of the body force $\left(v_{t}=v_{t 1}\right)$, (3.5) becomes:

$$
0=\frac{1}{r} \frac{\partial}{\partial r}\left[\frac{r}{R e}\left(\left(1+v_{t 1}\right) \frac{\partial\left(\bar{u}_{z}\right)_{b}^{e}}{\partial r}\right)\right]+f,
$$

where $v_{t 1}$ is the eddy viscosity of the EPG reference flow (i.e. from the solution of (3.2)) and the superscript ' $e$ ' indicates that the values of $\left(\bar{u}_{z}\right)_{b}$ computed are estimates or predictions based on the above assumption about the turbulent viscosity. In addition, we can estimate the turbulent shear stress due to the perturbation flow from

$$
\left(\overline{u_{z}^{\prime} u_{r}^{\prime}}\right)_{b}^{e}=-\frac{v_{t 1}}{R e} \frac{\partial\left(\bar{u}_{z}\right)_{b}^{e}}{\partial r} .
$$

Consequently, with the availability of the solution of EPG reference flow (and hence $v_{t 1}$ ), the perturbation flow due to the imposed body force can be estimated by simple integrations of (3.6). In essence, the hypothesis introduced earlier in this section implies: (i) $\left(\bar{u}_{z}\right)_{b} \approx\left(\bar{u}_{z}\right)_{b}^{e}$ or $\bar{u}_{z} \approx\left(\bar{u}_{z}\right)_{p}+\left(\bar{u}_{z}\right)_{b}^{e}$, and, (ii) $\left(\overline{u_{z}^{\prime} u_{r}^{\prime}}\right)_{b} \approx\left(\overline{u_{z}^{\prime} u_{r}^{\prime}}\right)_{b}^{e}$ or $\overline{u_{z}^{\prime} u_{r}^{\prime}} \approx\left(\overline{u_{z}^{\prime} u_{r}^{\prime}}\right)_{p}+\left(\overline{u_{z}^{\prime} u_{r}^{\prime}}\right)_{b}^{e}$. That is, the body-force-influenced flow can be approximated by the sum of its corresponding EPG reference flow (i.e. an 'undisturbed' flow) and a perturbation flow due to the body force. The latter is readily computed from the body-force distribution and the EPG reference flow parameter $v_{t 1}$.

Integrating equation (3.1) results in an expression for the force balance of the flow:

$$
-\frac{r}{2} \frac{\mathrm{d} P}{\mathrm{~d} x}+\frac{1}{r} \int_{0}^{r} r f \mathrm{~d} r=\overline{u_{z}^{\prime} u_{r}^{\prime}}-\frac{1}{R e} \frac{\partial \bar{u}_{z}}{\partial r} .
$$

The first and second terms on the left-hand side are the contributions to the total shear stress from the pressure gradient and the body force, denoted as, $\tau_{p}$ and $\tau_{b}$, respectively. Letting $r=1$, we obtain the total wall shear stress to be $-(1 / \operatorname{Re})\left(\partial \bar{u}_{z} / \partial r\right)_{r=1}=-(1 / 2)(\mathrm{d} P / \mathrm{d} x)+\int_{0}^{1} r f \mathrm{~d} r$, that is, the total wall shear $\left(\tau_{w}\right)$ is made of contributions from the pressure force $\left(\tau_{w p}\right)$ and the additional non-uniform body force $\left(\tau_{w b}\right)$. Taking case B2 as an example, the total shear stress $(\tau)$ of the body-force-influenced flow together with those of its corresponding EFR and EPG reference flows are shown in figure 9 to illustrate a number of concepts 


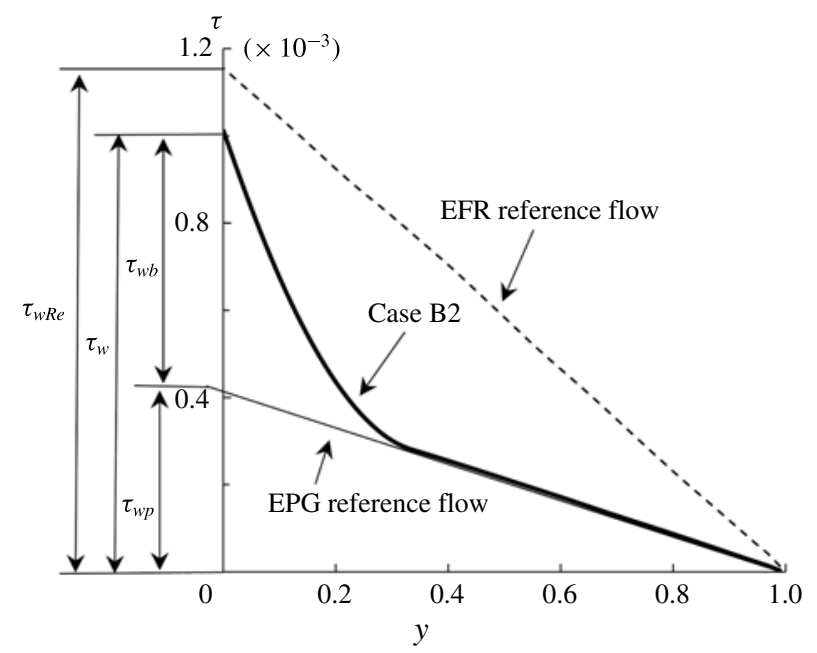

FIGURE 9. Total and wall shear stresses of case B2 and those of its corresponding EFR and EPG reference flows.

introduced herein. In this particular case, the total wall shear $\left(\tau_{w}\right)$ is smaller than that of the EFR reference case $\left(\tau_{w R e}\right)$, but the opposite is true in some other cases. By definition, the wall shear stress of the EPG reference case and the contribution of the pressure gradient in the body-force-influenced case are equal, being $\tau_{w p}$. We define a friction velocity based on the pressure gradient component of the wall shear as $u_{\tau p}^{*}=\sqrt{\tau_{w p}^{*} / \rho^{*}}$, which is referred to as the apparent friction velocity, and similarly, $R e_{\tau p}=u_{\tau p}^{*} R^{*} / \nu^{*}$ as the apparent Reynolds number of the body-force-influenced flow. These are important parameters in the new framework of analysis. The values of the apparent Reynolds number for the various cases are shown in table 2, together with the averaged body force $(F)$ and the bulk velocity of the body-force-induced perturbation flow, $U_{b f}$.

We now inspect the turbulent viscosity obtained for the various test cases studied herein, which are shown in figure $10(a, b)$ in wall coordinates based on the conventional friction velocity $\left(u_{\tau}^{*}\right)$ and the apparent friction velocity $\left(u_{\tau p}^{*}\right)$, i.e. $y^{+}=\left(\sqrt{\tau_{w}^{*} / \rho^{*}} y^{*}\right) / v^{*}$ and $y^{+1}=\left(\sqrt{\tau_{w p}^{*} / \rho^{*}} y^{*}\right) / v^{*}$, respectively. It can be seen from figure $10(a)$ that the turbulent viscosity reduces in the body-force-influenced flows in comparison with the EFR reference flow. Generally speaking, the larger the body force, the stronger the reduction, which is consistent with the observations on turbulence quantities presented earlier. This is also in good accordance with the conventional theory, which explains flow laminarising phenomena (Launder \& Jones 1969; Sreenivasan 1982; Piomelli \& Yuan 2013).

By contrast, when the turbulent viscosity is plotted against $y^{+1}$ (figure $10 \mathrm{~b}$ ), the spread of the data of the various cases is much smaller in the wall region, say, $y^{+1}<40$, and much of the spread can be accounted for by the effect of the Reynolds number on the distribution of turbulent viscosity. To verify this idea, undisturbed flows close to the apparent Reynolds numbers of the various flow cases (and hence serving as the EPG reference flows) have been performed and the distributions of the turbulent viscosity of these cases are shown in figure $10(b)$ for direct comparison. It can be seen that the values of the turbulence viscosity in the various cases are largely bounded by those of the flows of $R e_{\tau}=110$ and 180. A closer study of the data reveals that cases 

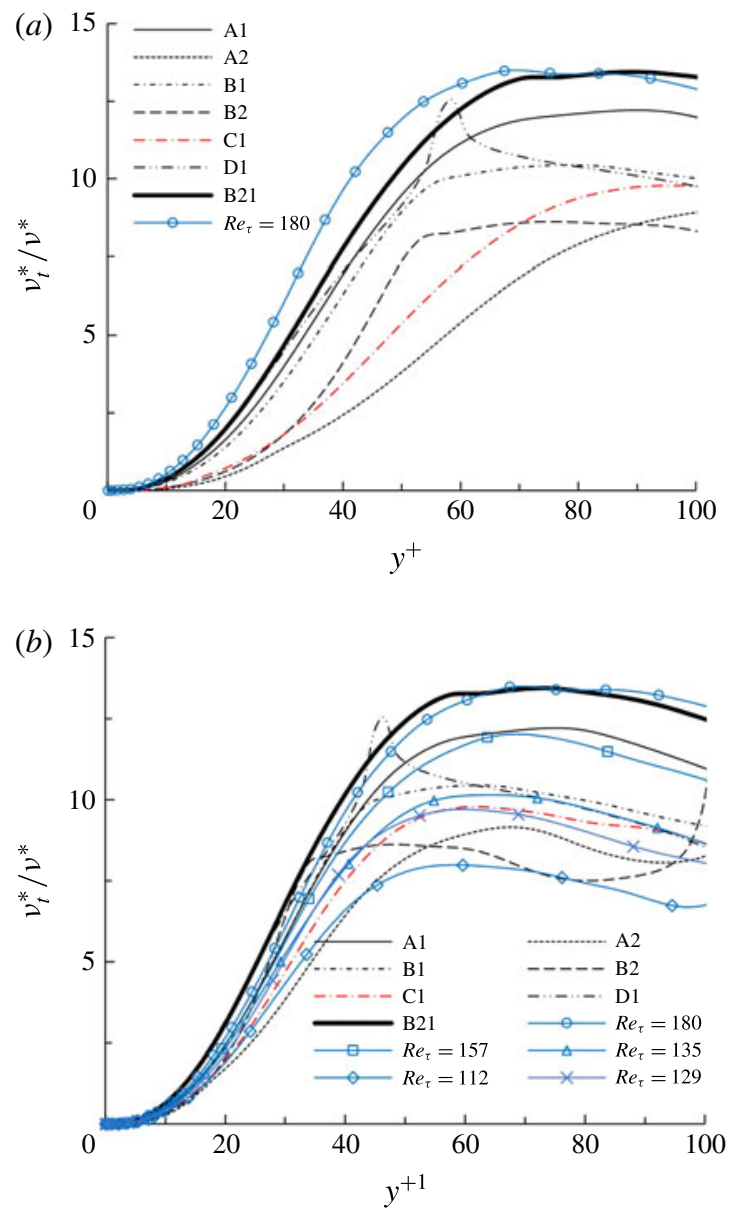

FIGURE 10. (Colour online) Distribution of turbulent viscosity in wall distance based on (a) conventional friction velocity and $(b)$ apparent friction velocity.

A1, A2, C1 and B21 agree closely with their corresponding EPG reference flows. The values of $v_{t}$ of B1, B2 and D1 overshoot those of their corresponding reference flows at some locations near the wall. These deviations are still relatively small considering the large range of flow conditions covered and the severity of the flow laminarisation observed. In conclusion, these results provide a good support for the hypothesis presented early: the values of the turbulent viscosity of the body-force-influenced flows is not significantly influenced by the body force and they agree well with those of their corresponding EPG reference flows. Consequently, the body-force-influenced flow in effect behaves as an 'undisturbed' flow at a lower apparent Reynolds number.

It is noted that in pursuing the development of semi-empirical correlations for buoyancy-influenced heat transfer, Jackson \& Hall (1979) introduced an assumption that the effect of buoyancy on the flow is to reduce the near-wall shear stress and hence turbulence production. They used the turbulent shear stress away from the buoyancy-influenced region to define a new equivalent flow, based on which heat transfer was calculated. This approach allowed them to develop a very successful, widely used, heat transfer correlation. The observation described herein, to some extent, explains the reasons behind the success. 


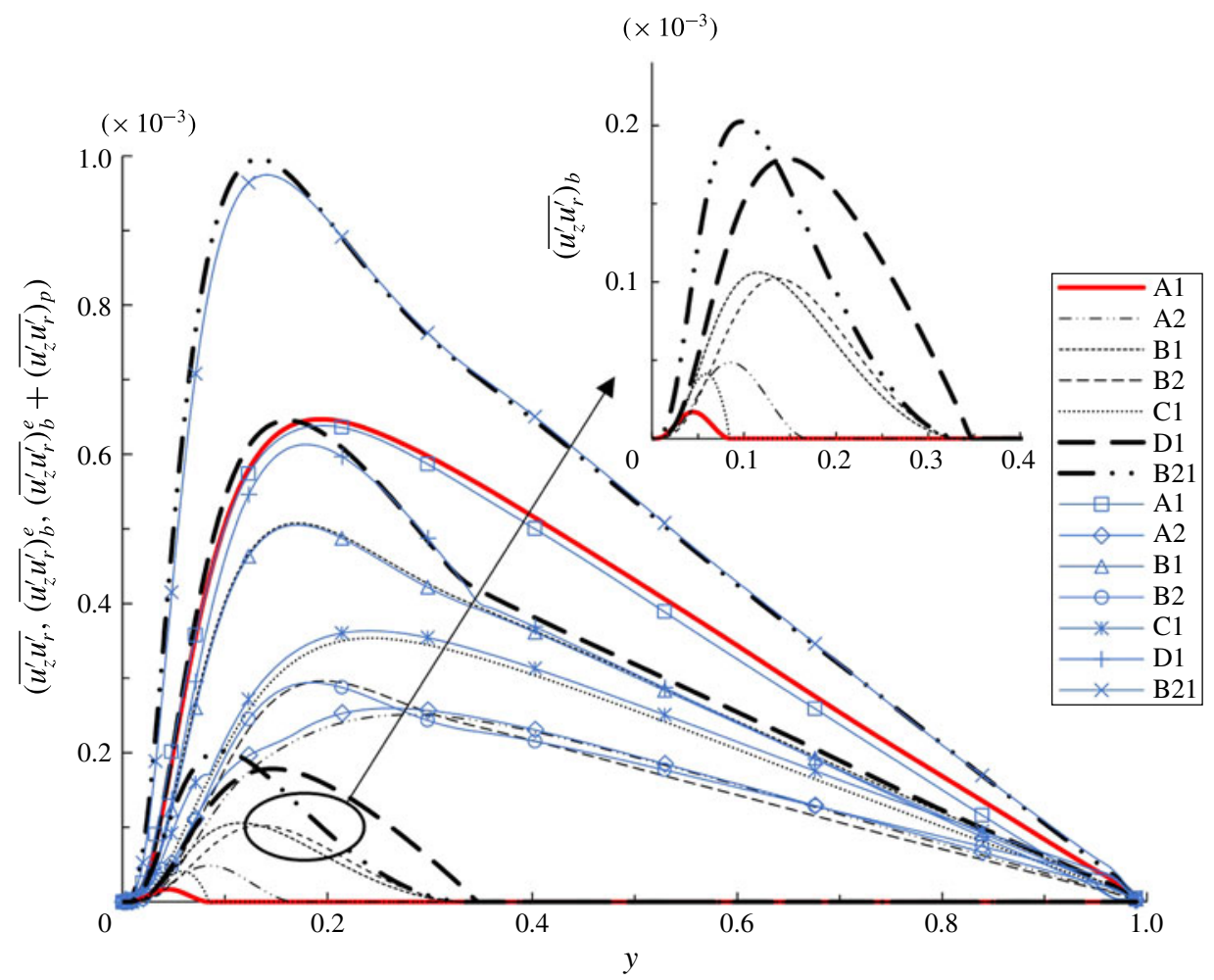

FIGURE 11. (Colour online) Distribution of turbulent shear stresses, (i) DNS results $\overline{u_{z}^{\prime} u_{r}^{\prime}}$ (lines only in main figure), (ii) estimated turbulent shear stress due to body force calculated from (3.7), $\left(\overline{u_{z}^{\prime} u_{r}^{\prime}}\right)_{b}^{e}$, (lines only in inset) and (iii) estimated overall turbulent shear stress using EPG reference flow data, $\left(\overline{u_{z}^{\prime} u_{r}^{\prime}}\right)_{p}+\left(\overline{u_{z}^{\prime} u_{r}^{\prime}}\right)_{b}^{e}$ (lines with markers).

To further elucidate the idea, next we study the turbulent shear stresses. Figure 11 shows three sets of data: (i) DNS results $\overline{u_{z}^{\prime} u_{r}^{\prime}}$, (ii) estimated turbulent shear stress resulting from body force calculated from (3.7), $\left(\overline{u_{z}^{\prime} u_{r}^{\prime}}\right)_{b}^{e}$ and (iii) estimated overall turbulent shear stress using EPG reference flow data, $\left(\overline{u_{z}^{\prime} u_{r}^{\prime}}\right)_{p}+\left(\overline{u_{z}^{\prime} u_{r}^{\prime}}\right)_{b}^{e}$. It is noted that since the values of $R e_{\tau p}$ of some cases are noticeably different from the closest $R e_{\tau}$ of the available 'standard' cases, the EPG data used here are based on an interpolation of results with the closest $R e_{\tau}$. It can be seen that the body-force-induced turbulent shear stress, $\left(\overline{u_{z}^{\prime} u_{r}^{\prime}}\right)_{b}^{e}$, is only limited to the near-wall region, and the values vary significantly from case to case. Interestingly, the magnitude of $\left(\overline{u_{z}^{\prime} u_{r}^{\prime}}\right)_{b}^{e}$ is not directly corresponding to the averaged body force $(F)$. For example, the body forces of A1 and B1 are both 0.35 , but $\left(\overline{u_{z}^{\prime} u_{r}^{\prime}}\right)_{b}^{e}$ is significantly higher in B1 than in A1. Clearly this difference stems from the different coverages of the body force in the two flows. The larger the coverage, the larger the value of $\left(\overline{u_{z}^{\prime} u_{r}^{\prime}}\right)_{b}^{e}$. In fact, the contribution of $\left(\overline{u_{z}^{\prime} u_{r}^{\prime}}\right)_{b}^{e}$ to the total turbulent shear stress in A1 is so small that it can be neglected. This has an interesting implication, that is, the body-force-induced perturbation flow can be seen to be a laminar flow, and that the turbulent shear stress of the body-force-influenced flow is the same as that of the corresponding EPG reference flow. It is particularly interesting to note that the total body force does not have to be small; it only requires the coverage to be small. Flows that are in this category are A1, A2 and C1. Typically, 

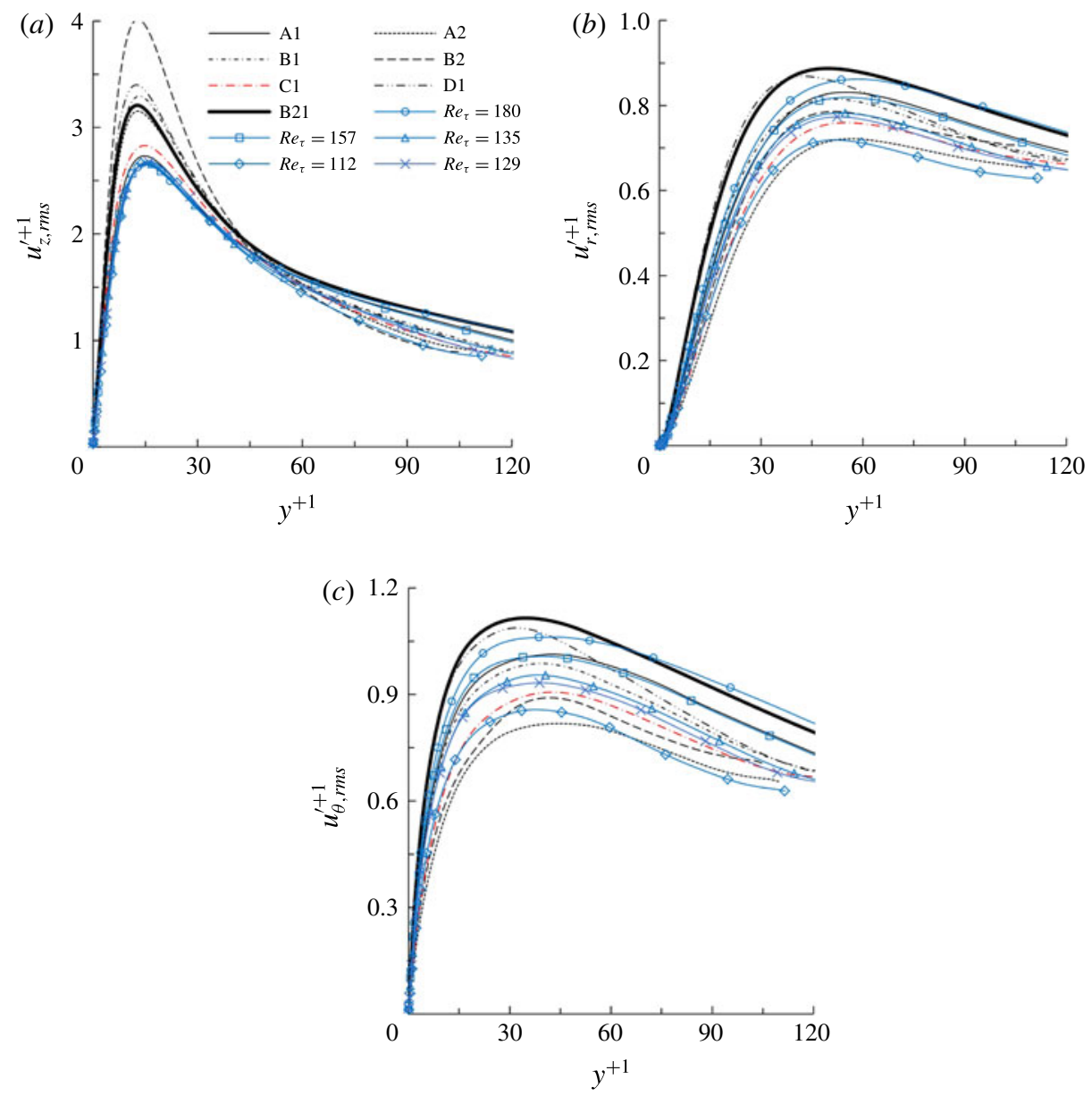

FIGURE 12. (Colour online) Distribution of the root-mean-square turbulent velocities normalised by the apparent friction velocity.

the body-force-induced turbulence shear stress $\left(\overline{u_{z}^{\prime} u_{r}^{\prime}}\right)_{b}^{e}$ will be negligible when the coverage of the body force is limited $y^{+}<20$, which is the case in many mixed convection flows. To facilitate discussion, we refer to them as low $\left(\overline{u_{z}^{\prime} u_{r}^{\prime}}\right)_{b}^{e}$ flow cases.

We now compare the turbulent shear stress directly calculated from the DNS results of the various cases $\left(\overline{u_{z}^{\prime} u_{r}^{\prime}}\right)$ and those estimated using the EPG reference flows, i.e. $\left(\overline{u_{z}^{\prime} u_{r}^{\prime}}\right)_{p}+\left(\overline{u_{z}^{\prime} u_{r}^{\prime}}\right)_{b}^{e}$. The agreement between the two sets of data is strikingly close in most cases. For the low $\left(\overline{u_{z}^{\prime} u_{r}^{\prime}}\right)_{b}$ cases, this implies that the turbulent shear stress is exactly the same as that of the EPG reference flow and the body force does not change it in any way. For other cases, it can be inferred that the perturbation flow induced by the body force does result in an additional turbulent shear stress, but its value can be estimated with the turbulent viscosity of the EPG reference flow. Overall these results again provide evidence supporting the underlining assumption of the theory proposed herein, that is, the turbulence mixing characteristics are not modified by the body force.

Next we study the turbulent normal stresses in the new framework. The stresses normalised by the apparent frictional velocity $\sqrt{\tau_{w p}^{*} / \rho^{*}}$ are plotted against $y^{+1}$ in figure 12. Before studying the detailed behaviour, we first note that the apparent 
Reynolds numbers of most flows studied herein are rather low, and some of the flows are in the transitional flow regime, refer to table 2. Tsukahara et al. (2014) studied low Reynolds number turbulent and transitional flows in a channel with $\operatorname{Re}_{\tau}$ between 64 and 180. They have shown that the peak root-mean-square values of the $u_{r}^{\prime}$ and $u_{\theta}^{\prime}$ at low Reynolds numbers reduce significantly. The DNS of low Reynolds number flows performed herein $\left(R e_{\tau}=112,129,135,157\right.$ and 180) clearly show this trend. These data serve as the EPG reference flows for the various flow cases (again refer to table 2). In strong contrast with figure 8 where the data are normalised with the conventional friction velocity, the spreads of $u_{r, r m s}^{\prime+1}$ and $u_{\theta, r m s}^{\prime+1}$ between the different test cases are small, and most of these can be accounted for by the Reynolds number effect. Close to the wall $\left(y^{+1}<30\right)$, the profiles of $u_{r, r m s}^{\prime+1}$ and $u_{\theta, r m s}^{\prime+1}$ follow very much what expected at their respective apparent Reynolds number in all the cases except for case D1, where the values are clearly higher than that of $R e_{\tau}=135$. The peak values also agree well with those of their corresponding EPG reference flows in most cases, except for cases B2 and D1, in which the peak values are higher than expected but are still within $10 \%$. These results demonstrate that, in contrast to the conclusions drawn earlier from the conventional approach using wall units based on the total flow, which show a strong reduction in turbulence, the radial and circumferential turbulence components are not significantly influenced by the imposed body force in the new framework.

In contrast to the trends exhibited in $u_{r, r m s}^{\prime+1}$ and $u_{\theta, r m s}^{\prime+1}$ (and that demonstrated in the conventional plots shown in figure 8), the peak values of the streamwise turbulent velocity are increased in all the test cases except for A1; in some cases, significantly. This is despite that the peak values of the corresponding EPG reference flows collapse on top of each other. We demonstrate later that the increase of $u_{z, r m s}^{\prime+1}$ is associated with the enhancement of the streaks in the flow resulting from the imposition of the body force. Referring to the turbulence regeneration cycle (Kim 2011), it appears that the body force enhances the linear processes of streak generation and the transient growth processes (the first and second legs) through the boundary layer that it induces. It does not seem to significantly influence the nonlinear vortex formation (the third leg). This will be further discussed later with reference to the flow structures.

Figures 13 to 16 show the budget terms of the streamwise and wall-normal stresses normalised by $u_{\tau}^{* 4} / \nu^{*}$ and $u_{\tau p}^{* 4} / \nu^{*}$, respectively. As expected, the terms for both $u_{z}^{\prime}$ and $u_{r}^{\prime}$ normalised based on $u_{\tau}^{*}$ reduce in all the test cases, and in many cases, such as $\mathrm{A} 2$, B2 and $\mathrm{C} 1$, the reduction is very significant. This is consistent with the traditional view of flow laminarisation, demonstrating that turbulence is suppressed due to the presence of the body force. The picture is however very different when the terms are normalised based on $u_{\tau p}^{*}$. For the streamwise component, the budget terms in A1 and $\mathrm{C} 1$ agree very well with their corresponding reference values. For the rest of the cases, both the production and the dissipation are increased by various amounts. This trend is consistent with the observations in figure 12 where $u_{z, r m s}^{\prime+1}$ is presented. The increase of the production is largely related to the generation of elongated streaks. For the wall-normal component, there is no direct production. The supply of energy comes from the pressure strain term, whereas the sink is the dissipation. In addition, the pressure diffusion term is also significant, especially close to the wall. It can be seen from figure 16 that, overall, the terms in the body-force-influenced flows agree very well with those of the base case, especially considering the large deviations shown in the conventional presentation (figures 13 and 14). The agreements between the main terms (pressure strain and dissipation) are particularly good for A1 and B1. This statement is also largely true for $\mathrm{A} 2$ and $\mathrm{C} 1$, though the pressure strain and 


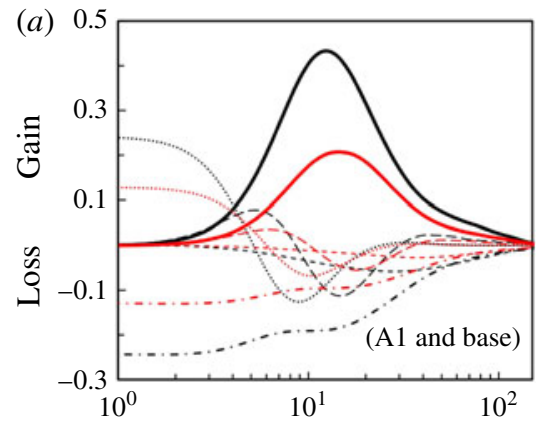

(b)
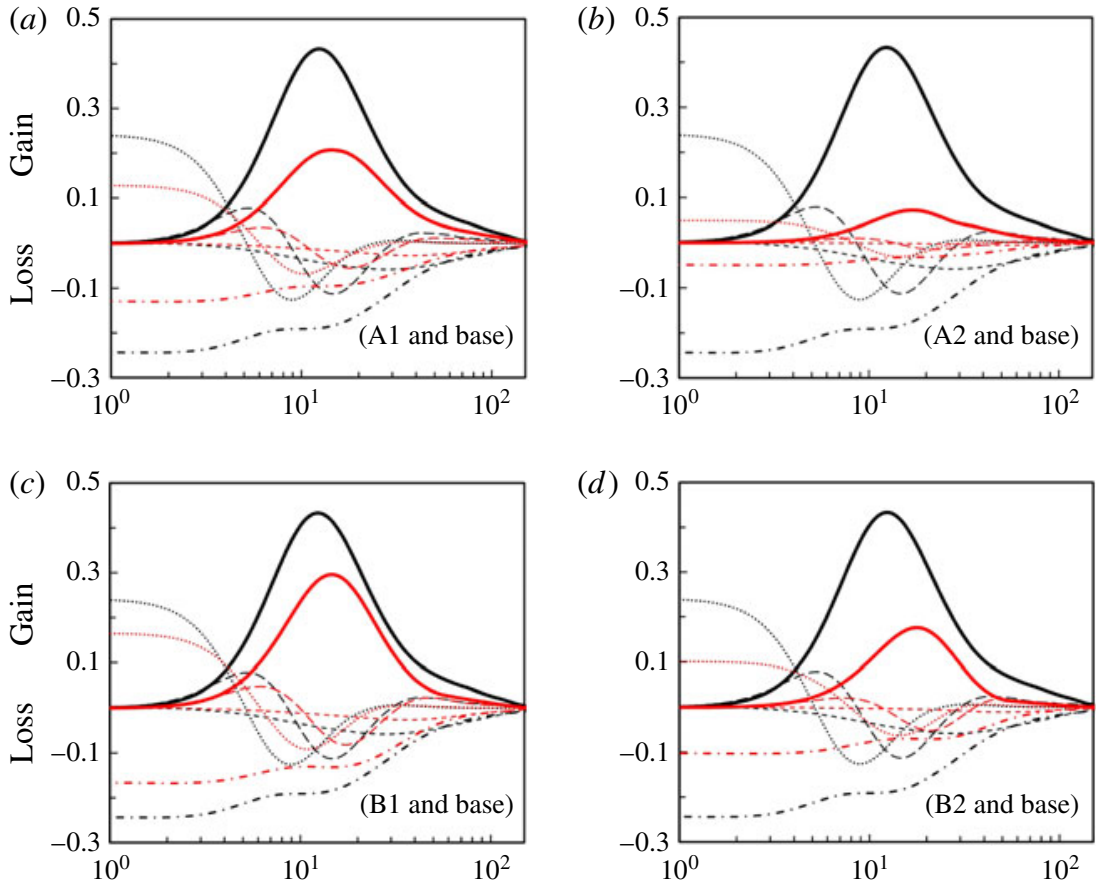

(d)
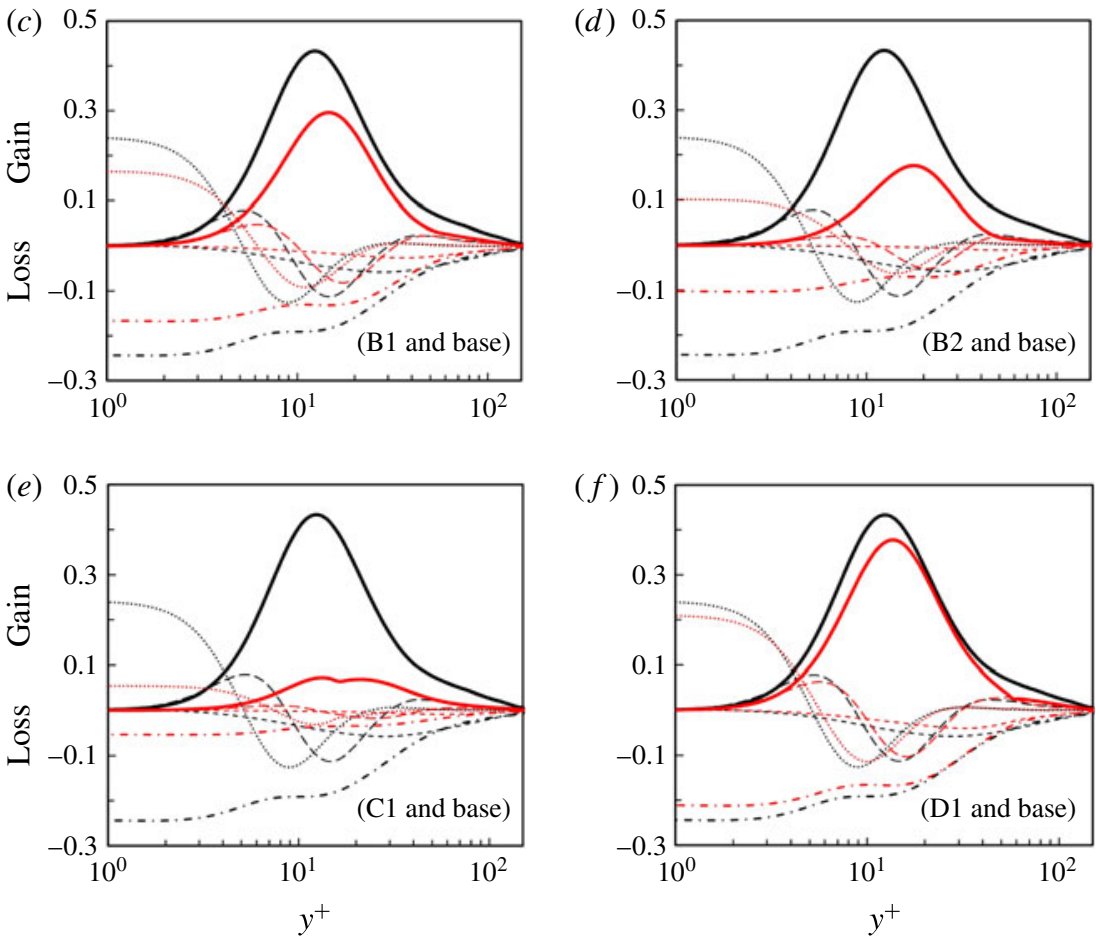

FIgURE 13. (Colour online) The budget terms of the streamwise turbulent fluctuating velocity in conventional wall units. Solid lines: production; chain dotted lines: dissipation; short dashed lines: pressure strain; long dashed lines: turbulent transport; dotted lines: viscous diffusion. Thick lines: base case $\left(R e_{\tau}=180\right)$; thin lines: body-force-influenced cases.

dispersion terms show an interesting strong reduction near the wall $\left(y^{+1}<8\right)$. Both terms agree reasonably well with their respective reference data in the core region. Another observation is that the source term, pressure strain, increases somewhat in several cases, including B21 (not shown), B2 and D1, and to a lesser extent B1. These are the cases where the body-force-induced turbulent shear stress is relatively large. Overall however the agreement between the body-force-influenced cases and the reference cases is remarkable considering that in many cases the absolute values reduce by several folds. 


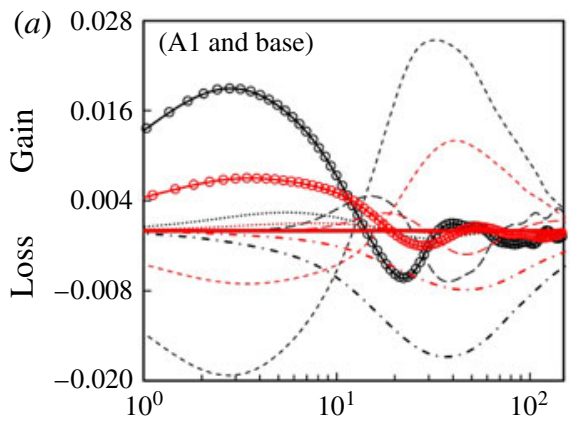

(b)
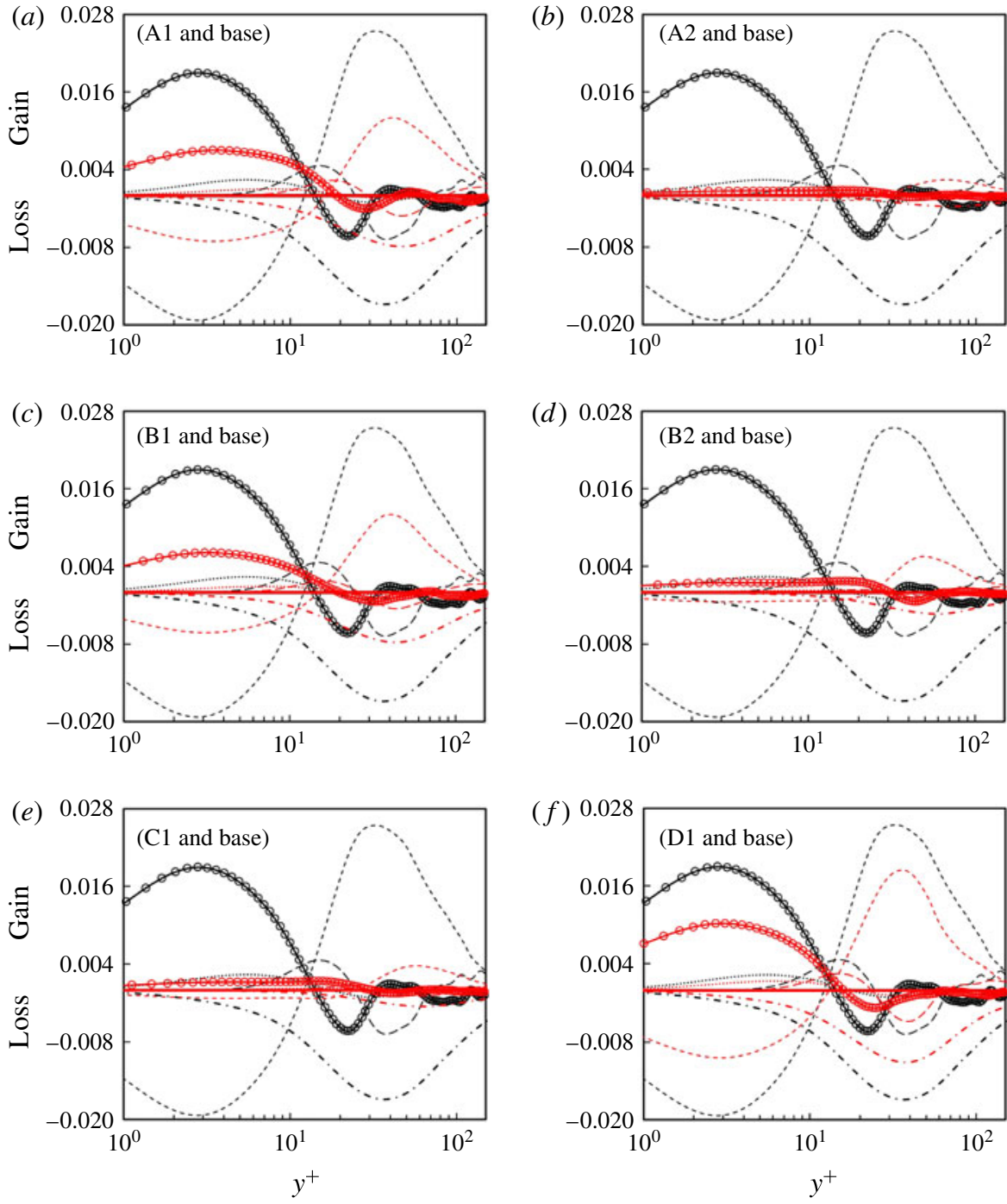

FIGURE 14. (Colour online) The budget terms of the wall-normal turbulent fluctuating velocity in conventional wall units. Solid lines with markers: pressure diffusion; chain dotted lines: dissipation; short dashed lines: pressure strain; long dashed lines: turbulent transport; dotted lines: viscous diffusion. Thick lines: base case $\left(\operatorname{Re}_{\tau}=180\right)$; thin lines: body-force-influenced cases.

Before leaving this section, we revisit some interesting features of the mean velocity field. Figure 17(a) shows the profiles of the mean velocity of the perturbation flow induced by the body force calculated from (3.6), $\left(\bar{u}_{z}\right)_{b}^{e}$; and figure $17(b, c)$ show the velocity difference, $\left(\bar{u}_{z}\right)^{\wedge}=\bar{u}_{z}-\left(\bar{u}_{z}\right)_{b}^{e}$, in outer scaling and wall scaling respectively. According to the theory established herein, $\left(\bar{u}_{z}\right)^{\wedge}$ of any flow case is expected to be in good agreement with the velocities of its corresponding EPG reference flows $\left(\bar{u}_{z}\right)_{p}$, which are also shown in the figure comparison. It can be seen from figure $17(b)$ that this is indeed the case for most flow cases. Similar to the turbulence quantities presented above, relatively larger discrepancies are observed in cases B2 and D1, 


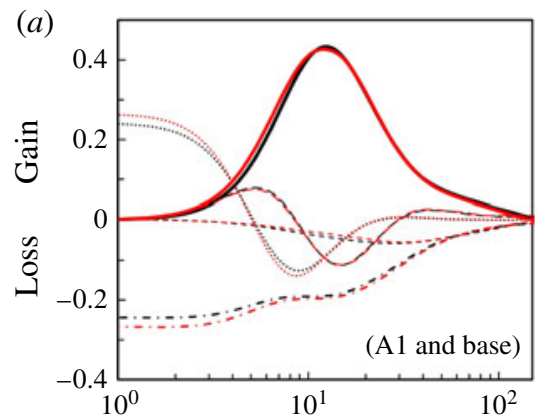

(b)
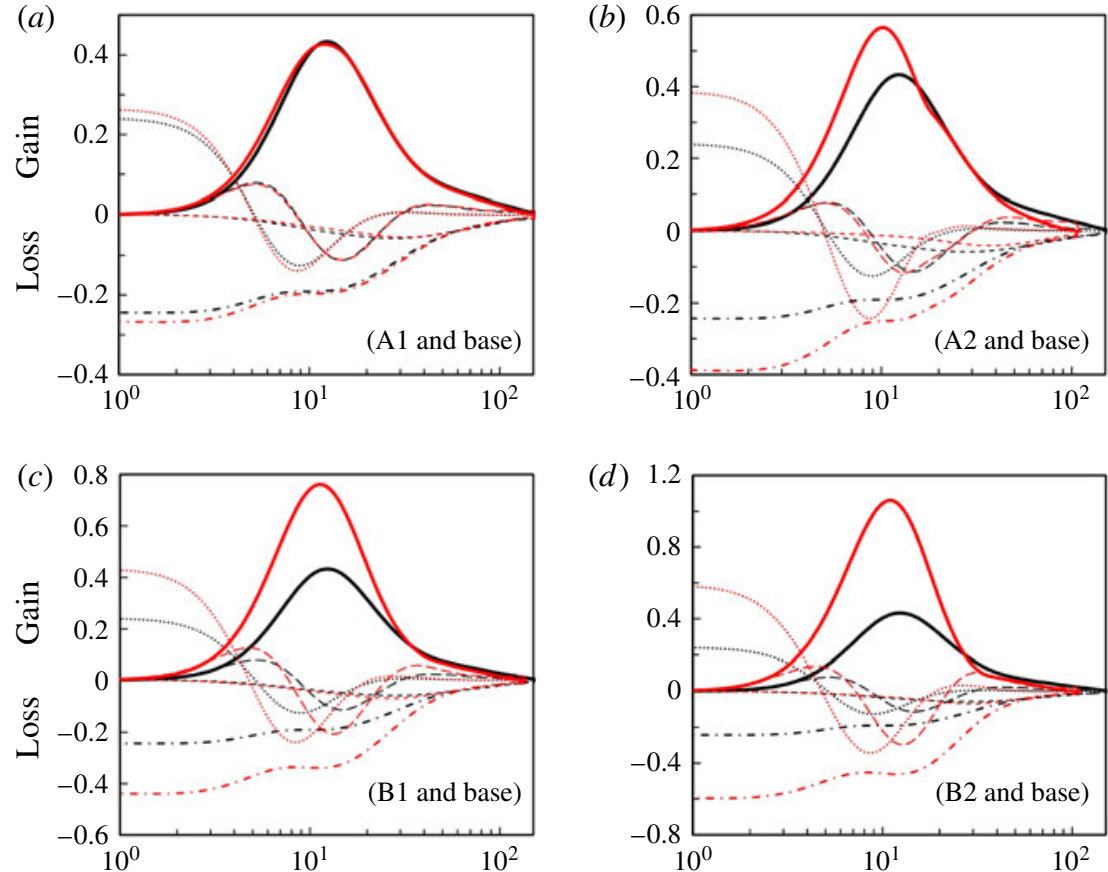

(d)
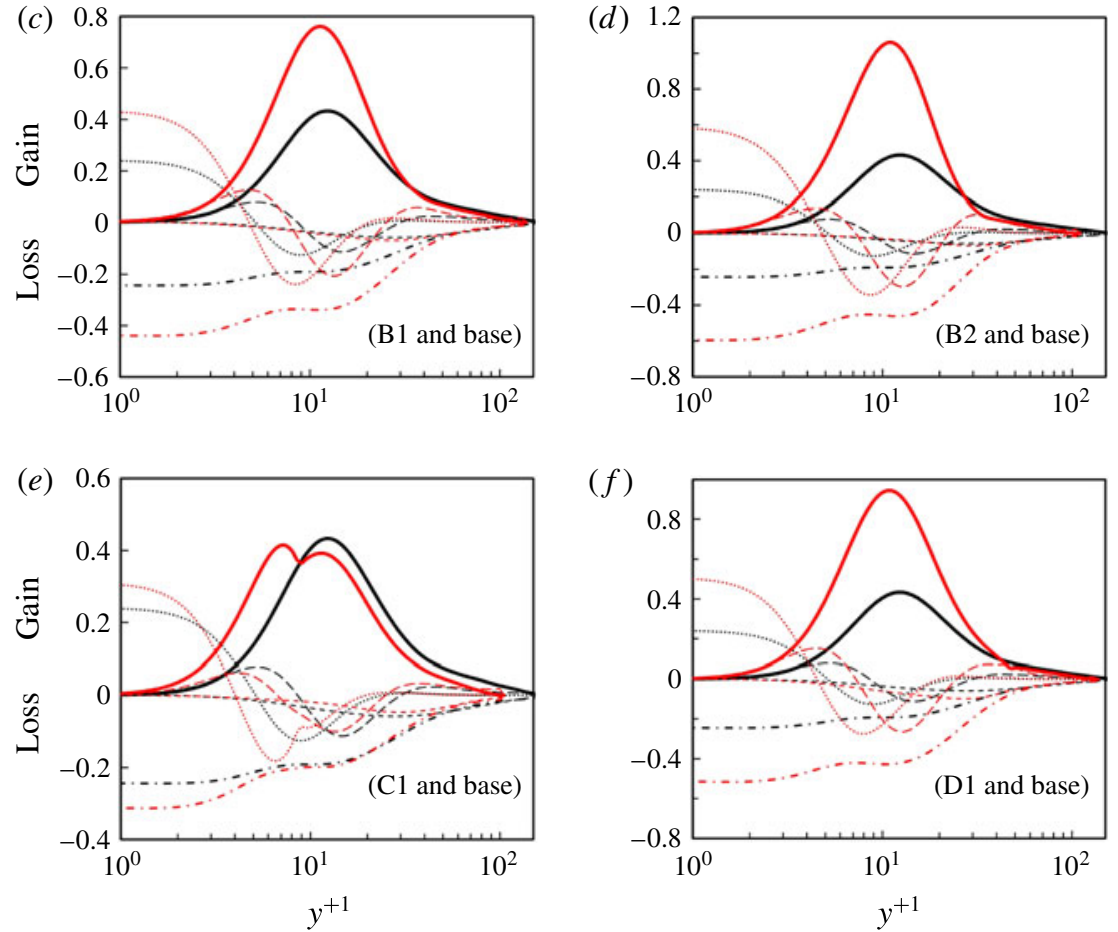

FIgURE 15. (Colour online) The budget terms of the streamwise turbulent fluctuating velocity in wall units based on the apparent friction velocity. See figure 13 for legend.

which are approximately $10 \%$. Next, it is interesting to note that the body-force perturbation flow is not necessarily directly proportional to the total body force $(F)$. The coverage has a significant effect. For example, A1 and B1 have the same total body force, but the perturbation flow of B1 is more than doubled that in A1. The next point to note is that the body-force-induced perturbation flow is very significant in some cases in comparison with the EPG reference flows. The ratios between the two flows are $13 \%$ for A1, approximately $35 \%$ for B1 and B21, approximately $50 \%$ for $\mathrm{C} 1$ and $\mathrm{D} 1$ and then $70 \%$ and $85 \%$ for A2 and B2. Apparently, the addition of such a large perturbation flow does not seem to have a significant impact on the key turbulent characteristics of the flow as shown above. Finally it can be seen from the wall coordinates velocity plot that the profiles of $\left(\bar{u}_{z}\right)^{\wedge}$ agree well with the EFR reference flow profile in most of the cases, clearly exhibiting a region of logarithmic 

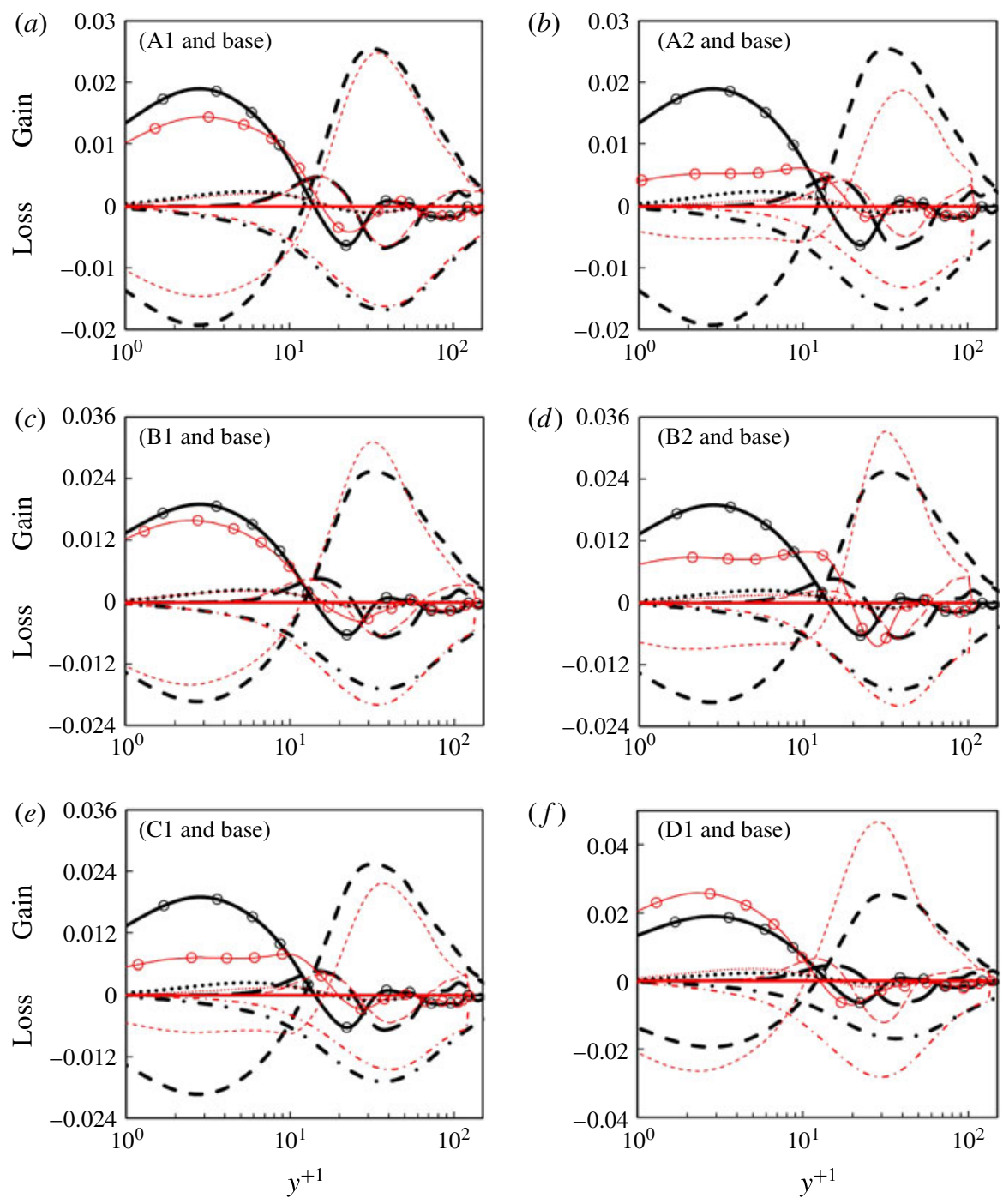

FIGURE 16. (Colour online) The budget terms of the wall-normal turbulent fluctuating velocity in wall units based on the apparent friction velocity. See figure 14 for legend.

behaviour. There are some cases where the velocity profile shows a relatively strong upshift, which is normally seen as a sign of laminarisation. This occurs in A2 and B2, which correspond to the lowest $\left(\bar{u}_{z}\right)^{\wedge}$ and therefore the corresponding EPG reference flows have the lowest $R e_{\tau}$.

\subsection{Friction factor and the FIK identity analysis}

Fukagata et al. (2002) introduced a simple but useful analysis of the contributions of different dynamic effects on the friction drag for turbulent flows. The local friction is decomposed into a laminar, turbulent, inhomogeneous and transient components. This method has been widely used in flow control and drag reduction investigations and is referred to as the FIK identity. For a flow in a pipe considered herein (which is 

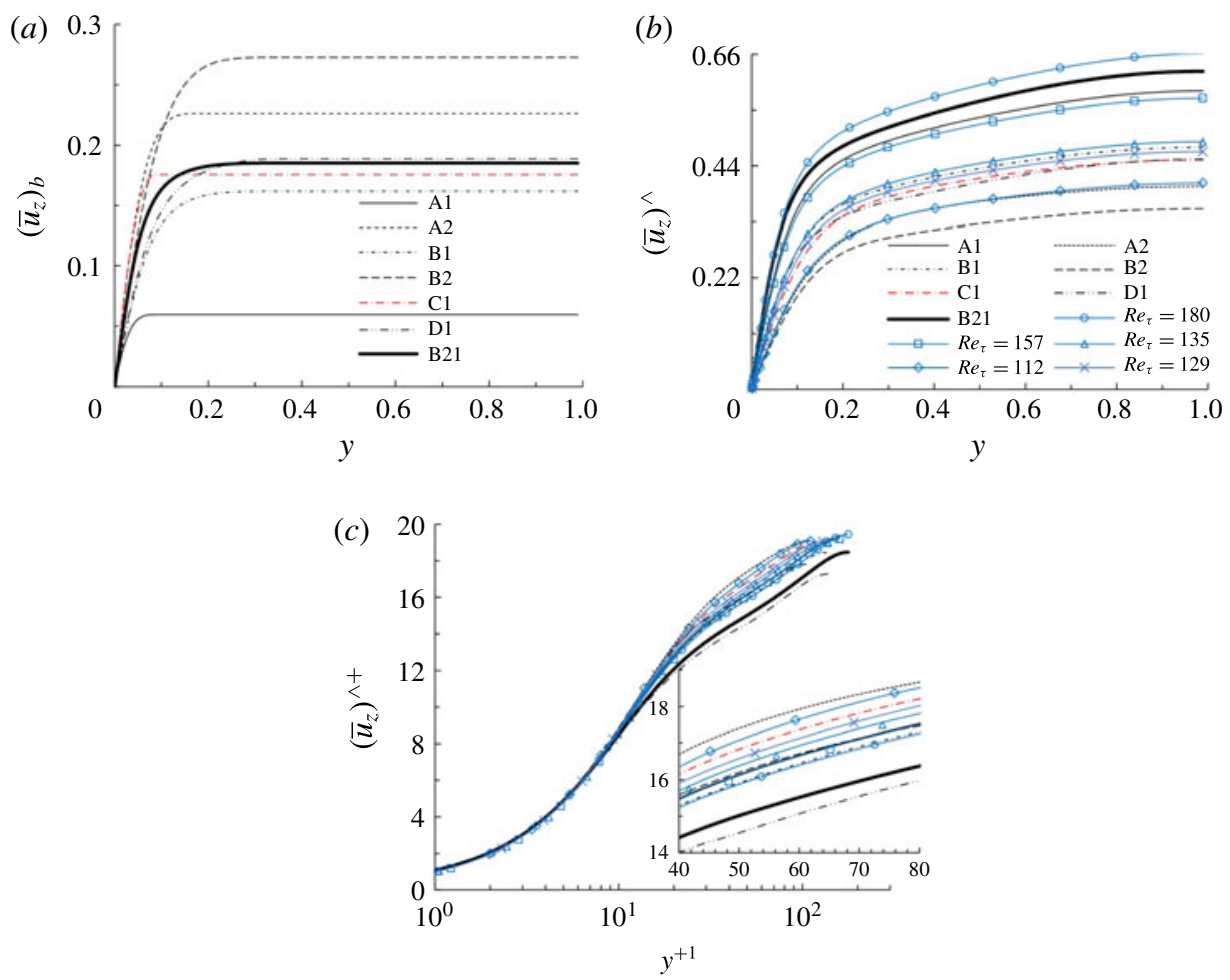

FIGURE 17. (Colour online) Velocity profiles. (a) Body-force-induced perturbation flow; (b) total flow subtracting the body-force-induced flow, together with EPG reference flows; and $(c)$ same as $(b)$ but in wall coordinates.

described by (3.1)), the FIK expression is (Fukagata et al. 2002),

$$
C_{f}=\frac{16}{R e}+16 \int_{0}^{1} 2 r \overline{u_{z}^{\prime} u_{r}^{\prime}} r \mathrm{~d} r+16 \int_{0}^{1}\left(r^{2}-1\right)(f-F) r \mathrm{~d} r,
$$

where $C_{f}=\tau_{w}^{*} /\left(\rho^{*} U^{* 2} / 2\right)=8 \tau_{w}^{*} /\left(\rho^{*} U_{P}^{* 2}\right)$, and $F=2 \int_{0}^{1} f r \mathrm{~d} r$, being the averaged body force. From left to right, the terms represent the contributions due to laminar and turbulent flows, and the body force. To better understand the contribution of the body force, the turbulent contribution is further split into (i) a component corresponding to the EPG reference flow, $\left(\overline{u_{z}^{\prime} u_{r}^{\prime}}\right)_{p}$, and (ii) one due to the perturbation flow induced by the body flow, which is calculated by subtracting the above component from the total turbulent shear stress, $\left(\overline{u_{z}^{\prime} u_{r}^{\prime}}\right)_{b}=\overline{u_{z}^{\prime} u_{r}^{\prime}}-\left(\overline{u_{z}^{\prime} u_{r}^{\prime}}\right)_{p}$.

$$
16 \int_{0}^{1} 2 r \overline{u_{z}^{\prime} u_{r}^{\prime}} r \mathrm{~d} r=16 \int_{0}^{1} 2 r\left(\overline{u_{z}^{\prime} u_{r}^{\prime}}\right)_{p} r \mathrm{~d} r+16 \int_{0}^{1} 2 r\left(\overline{u_{z}^{\prime} u_{r}^{\prime}}\right)_{b} r \mathrm{~d} r .
$$

The FIK analysis of the various laminarising cases is shown in figure 18. It can be seen that the total friction factor of the laminarising flows can be higher or lower than that of the EFR reference flow. The figure also illustrates that the contribution of the turbulence associated with the perturbation flow due to the body flow is negligible in $\mathrm{A} 1, \mathrm{~A} 2$ and $\mathrm{C} 1$ and is substantial in the other test cases. This is consistent with earlier 

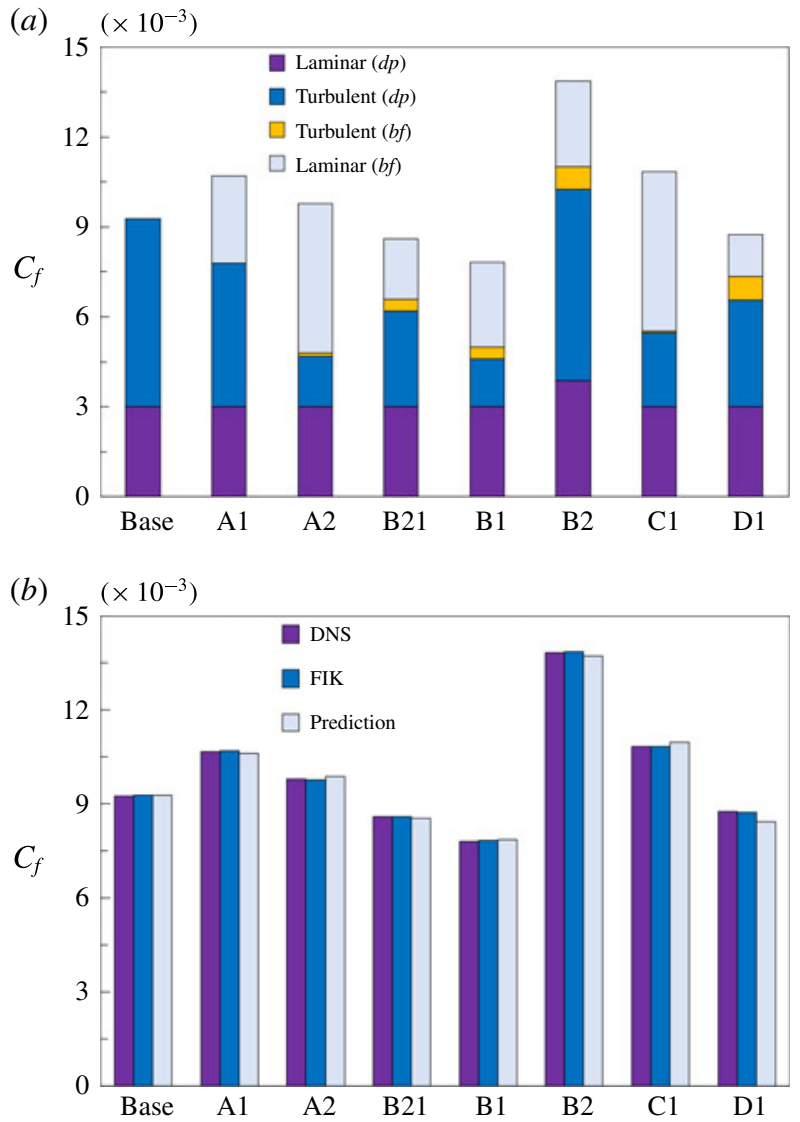

FIGURE 18. (Colour online) Friction coefficient. (a) Contributions of the various components of FIK analysis, laminar ( $d p$ and $b f)$ refer to the right-hand side terms 1 and 3 of (3.9), respectively; turbulent ( $d p$ and $b f)$ refer to the right-hand side terms 1 and 2 of (3.10), respectively; (b) comparison of friction coefficients calculated from velocity gradient (DNS), FIK analysis and predictions based on EPG reference flows.

observations (refer to figure $11(a)$ where $\left(\overline{u_{z}^{\prime} u_{r}^{\prime}}\right)_{b}$ is shown, for example). Comparing A1 with A2 (or B1 with B2), it is noted that the flow is more strongly laminarised as a result of a stronger body force, and hence a smaller contribution from $\left(\overline{\left(u_{z}^{\prime} u_{r}^{\prime}\right.}\right)_{p}$ in the former, but the contribution from the body force itself increases. If a reduction of the friction factor is the objective, increasing the amplitude of the body force is more effective than increasing the coverage, comparing, B1 and B2 with A1 and A2.

Next, we apply the theory established in $\$ 3.3$ to the prediction of the friction factors. We have established that the turbulent viscosity in the body-force-influenced flows remains little changed from the values of their corresponding EPG reference flows and that the turbulent shear stress due to the body-force-induced perturbation flow can be estimated using (3.7). Now, in (3.10), we replace $\left(\overline{u_{z}^{\prime} u_{r}^{\prime}}\right)_{b}$ with $\left(\overline{\overline{u_{z}^{\prime} u_{r}^{\prime}}}\right)_{b}^{e}$. The results are shown in figure 18(b) labelled 'Predictions'. It can be seen that these predictions are very close to those calculated from the original FIK formulation (3.9). Both agree very well with the friction factor calculated directly using the velocity gradient (labelled DNS in the figure). Consequently, the friction factor of a 
laminarising flow can be accurately predicted simply using an undisturbed turbulent flow data (the EPG reference flow) and the profile of the imposed body force.

\subsection{Full laminarisation}

Consider a turbulent flow at a prescribed Reynolds number subjected to a nonuniformly distributed body force. From the results presented in $\S 3.1$, we know that with the increase of the imposed body force while keeping the Reynolds number of the flow constant, the turbulence becomes progressively more strongly suppressed, that is, the flow is increasingly more severely laminarised. The terminology laminarisation refers to a comparison with the reference flow at the same Reynolds number. When the body force is sufficiently strong, the flow becomes fully laminarised, which is the case in A3 and B3. The above scenario occurs in many applications including mixed convection, for example, where the flow becomes progressively more severely laminarised with the increase of the buoyancy. The above process can be described using the new theory presented in $\S 3.3$. The body-force-influenced flow can be decomposed into a pressure-driven flow (i.e. the EPG reference flow) and a perturbation flow due to the body force. The former can be approximated by an undisturbed turbulent pipe flow, whereas the latter is a flow described by (3.6), which is dependent on the imposed body force and the EPG reference flow (through $v_{t}$ ). The bulk velocity of the EPG reference flow can be written as the difference between those of the total flow and the perturbation flow due to the body force, that is,

$$
U_{d p}^{*}=U^{*}-U_{b f}^{*} \quad \text { or } \quad U_{d p}=1 / 2-U_{b f} .
$$

With the increase of the body force $(F)$ while keeping the total flow constant, $U_{b f}$ increases and hence $U_{d p}$ reduces. There will be a point when the Reynolds number of the pressure driven flow is so low that it cannot sustain turbulence any longer, and hence the flow becomes laminar. This critical body force can be calculated using (3.11) given a critical Reynolds number of 2300, for example.

In the above discussion, the Reynolds number of the EPG reference flow plays an important role. Next we relate it to the imposed body force. Following the approach adopted by Fukagata et al. (2002), integrating equation (3.5) three times and making use of integration by parts, we can derive an expression for the mean velocity for the perturbation flow driven by the body force:

$$
U_{b f}=\operatorname{Re}\left[\frac{1}{2} \int_{0}^{1}\left(1-r^{2}\right) f r \mathrm{~d} r+\frac{1}{2} \int_{0}^{1} 2 r\left(\overline{u_{z}^{\prime} u_{r}^{\prime}}\right) b r \mathrm{~d} r\right] .
$$

Noting that $\operatorname{Re}_{d p}=\left(2\left(U^{*}-U_{b f}^{*}\right) R^{*}\right) / \nu^{*}=\operatorname{Re}\left(1-2 U_{b f}\right)$, we have

$$
\frac{R e_{d p}}{R e}=1-2 U_{b f},
$$

where $U_{b f}$ is calculated from (3.12). The above ratio of Reynolds numbers of the apparent and total flows effectively defines how far turbulence is reduced, or, the degree of laminarisation of the flow due to the imposition of the body force. This parameter is hence referred to as the laminarisation ratio. It is noted however that this parameter does not directly show how far the friction factor is varied, since the body force itself causes an additional friction to balance it, see discussion in $\S 3.4$. The total friction is the sum of the friction from the apparent flow and the integral 
of the body force. In addition, the laminarisation ratio does not tell if a flow is fully laminarised either. The criterion for laminarisation is that the Reynolds number of the apparent flow reduces to a degree when it cannot sustain turbulence any more, that is, $R e_{d p}<2300$. Combining this with (3.13), a criterion for full laminarisation is obtained,

$$
\operatorname{Re}\left(1-2 U_{b f}\right)<2300 .
$$

It is noted here that even though the parameter that is evaluated is the Reynolds number of the pressure-driven flow, the effect of the body force is directly reflected in the resultant equations (3.11) and (3.14). After all, the body force is the reason for the changes in the flow. In the literature, criteria for flow laminarisation, even based on advanced mechanistic models, require experimental data to tune coefficients. By contrast, the above criterion is simply based on that the apparent Reynolds number of the flow is below a laminar-turbulent transition Reynolds number, which is the only constant used.

For the low- $\left(\overline{u_{z}^{\prime} u_{r}^{\prime}}\right)_{b}$ cases, the second term of (3.12) is negligible and this normally occurs when the body force is limited to $y^{+}<20$, which can happen in various real flows. For ease of reference, the laminar-flow-only expression is given by:

$$
U_{b f}=\operatorname{Re}\left[\frac{1}{2} \int_{0}^{1}\left(1-r^{2}\right) f r \mathrm{~d} r\right] .
$$

We now evaluate the body-force-induced flow based on (3.15) for the linear and stepchange forces to establish some trends. Substituting (2.5) and (2.6) into (3.15), and integrating, the following are resulted for linear and step-change forces, respectively:

$$
\begin{gathered}
U_{b f}=\frac{R e}{120} \frac{a}{1-r_{0}}\left(-3 r_{0}^{5}+10 r_{0}^{3}-15 r_{0}+8\right), \\
U_{b f}=\frac{R e}{8} a\left(r_{0}^{4}-2 r_{0}^{2}+1\right) .
\end{gathered}
$$

Substituting the above into (3.14), we obtain the criteria for full flow laminarisation for linear and step distributions of body forces, respectively, under the condition when the body-force-induced flow perturbation causes negligible turbulent shear stresses:

$$
\begin{gathered}
a \operatorname{Re}^{2}\left[\frac{1}{60\left(1-r_{0}\right)}\left(-3 r_{0}^{5}+10 r_{0}^{3}-15 r_{0}+8\right)\right]>(\operatorname{Re}-2300), \\
a \operatorname{Re}^{2}\left[\frac{1}{4}\left(r_{0}^{4}-2 r_{0}^{2}+1\right)\right]>(\operatorname{Re}-2300) .
\end{gathered}
$$

Note that $a R e^{2}=\left(a^{*} R^{* 3}\right) /\left(\rho^{*} v^{* 2}\right)$. Consequently, the left-hand side of the above equations, which basically is the Reynolds number of the body-force-induced flow $\left(R e_{b f}\right)$, is independent of the bulk velocity of the flow, $U^{*}$. The above equation also implies that for both the linear and step distributions, the $R e_{b f}$ increases proportionally with the increase of the amplitude, whereas although increasing the coverage $r_{0}$ also increases $R e_{b f}$, the relationship is nonlinear.

It is worth noting that full flow laminarisation (reverse transition) is not an absolute, definitive event, but to some extent is dependent on the particular circumstances. The critical Reynolds number, 2300, used above is only a nominal value. In experiments, the flow can be laminar at a rather high Reynolds number if the experimental facility is free from disturbances and noise. In computer simulations, 
both the initialisation of the flow and the size of the computational domain influence the critical Reynolds number. In the study of Tsukahara et al. (2014), they have achieved turbulent/transitional flow in a channel at $R e_{\tau}=64$. In the present study, we have not attempted systematically increasing the body force to determine the critical flow. However, in groups A and B, the cases with the highest body force that still remain turbulent are cases A2 and B2. Their Reynolds numbers $(R e)$ are 3100 and 2862, (or for $R e_{\tau}, 112$ and 111), respectively. Cases A3 and B3 are evidently laminarised, but the corresponding body-force-driven flow is significantly greater than the critical value required to cause a full laminarisation.

\subsection{Flow structures}

The high- and low-speed streaks and the vortical cores of selected cases are visualised in figure 19 using isosurfaces of the fluctuating streamwise velocity $u^{\prime}= \pm 0.15$ and $\lambda_{2}=-1$, respectively. Comparing B1 and B2 with the EFR reference flow, it is clear that both the number of streaks and the number of the vortices reduce significantly due to the effect of the body force; and the stronger the body force, the more significant the reduction is. It is interesting to note however, that with the increase of the body force, the streaks appear to become longer. This is not surprising since, as we note before, the cases with a stronger body force corresponds to a EPG reference flow of a lower Reynolds number. It is known that streaks are longer in low Reynolds number flows. Next we note that as far as B21 is concerned, the base case $\left(R e_{\tau}=180\right)$ is its EPG reference flow. It is apparent that the numbers of vortical structures are largely the same in the two flows, whereas there are clearly more streaks in B21 than in the base case. This interesting observation is in good accordance with the statistics discussed earlier. The former can be related to the observation that the pressure strain term in the body-force-influenced flows remains largely unchanged from their corresponding EPG reference flows, and in turn $u_{r}^{\prime}$ and $u_{\theta}^{\prime}$ are largely unchanged. On the other hand, the increased number of streaks explains the increase in $u_{z}^{\prime}$, even though the vortical structures remain largely unchanged.

The concept of turbulence regeneration cycle has been established over the last couple of decades which successfully explains wall turbulence flow physics and, in some cases, the mechanisms of flow control and drag reduction. Figure 20 shows a much simplified sketch illustrating the main processes of the regeneration cycle reproduced from Kim (2011). In the first leg, streamwise vortices (primarily consisting of the wall-normal and spanwise velocities) interact with the mean shear $\left(\partial \bar{u}_{z} / \partial y\right)$ generating streaks. This process is often referred to as lift-up. The streaks generated grow as long as the shear is maintained (second leg). Later, the streaks break down due to instability. At the third leg, new vortices are formed again due to three dimensional disturbances. It is clear that the streamwise vorticity and streaks are most important factors in the turbulence regeneration cycle of a wall shear flow depicted in figure 20. Below we analyse the streamwise vorticity and the streaks in the various flows studied.

Figures 21(a) and (b) show the root-mean-square of the fluctuating streamwise vorticity in wall coordinates based on the total flow $\left(u_{\tau}^{*}\right)$ and the apparent friction velocity $\left(u_{\tau 1}^{*}\right)$, respectively. It can be seen from figure $21(a)$ that the vorticity normalised with $u_{\tau}^{*}$ reduces significantly in the various test cases. The reduction is most severe in A2, with the value reducing to less than a $1 / 4$ at $y^{+}=1$, and the peak away from the wall reducing to approximately $30 \%$ of the reference value. Consequently, the turbulence activities are significantly reduced in the various cases in comparison with the EFR reference flow. This observation is consistent 

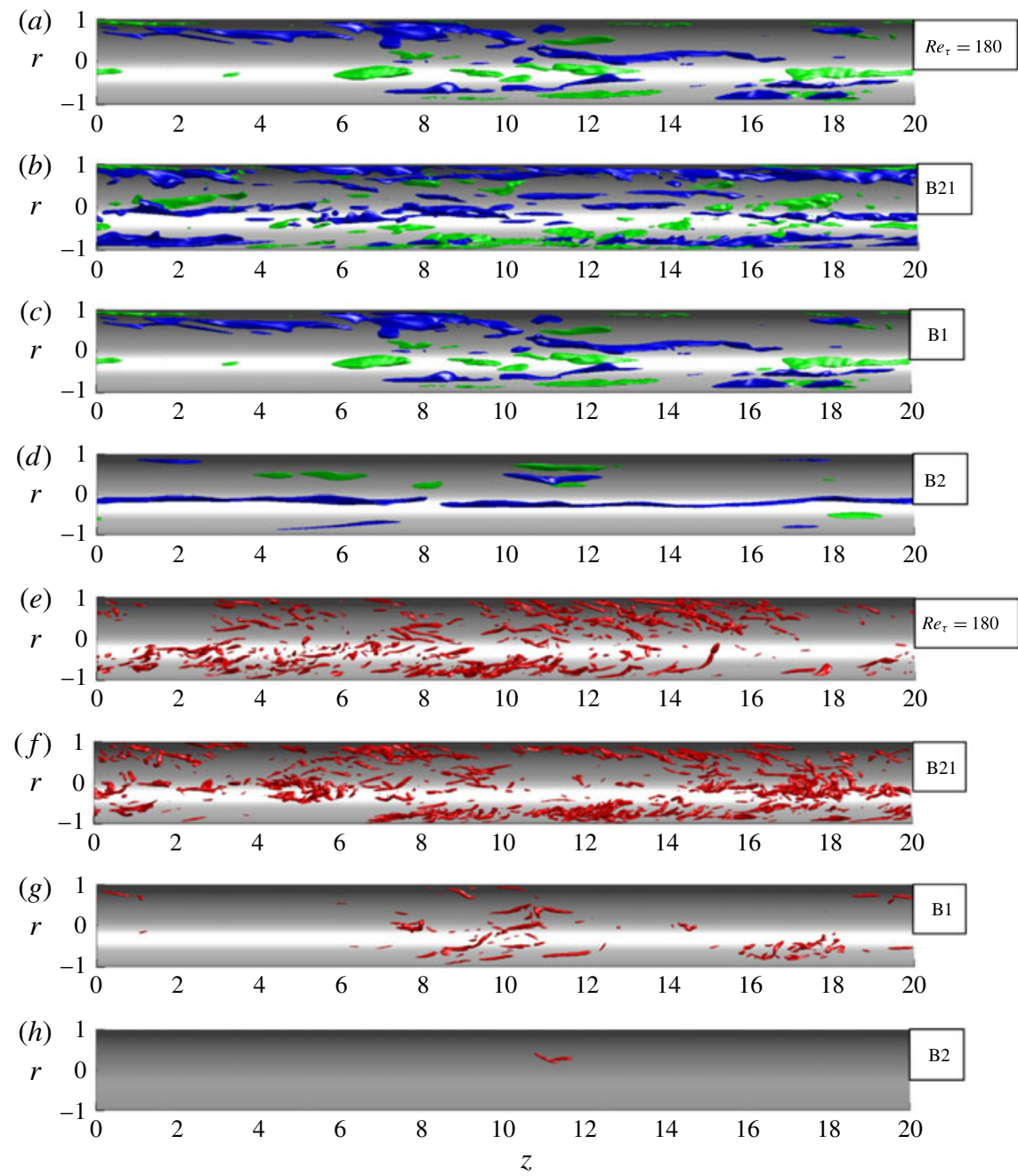

FIgURE 19. (Colour online) Turbulent structures in cases; base, B21, B1 and B2. $(a-d)$ High (green) and low (blue) speed streaks illustrated with three-dimensional isosurfaces of $u_{z}^{\prime}= \pm 0.15$ and $(e-h)$ bottom four panels: vortical structures illustrated with three-dimensional isosurfaces of $\lambda_{2}=-1.0$.

with the descriptions of laminarisation in various scenarios (Launder \& Jones 1969; Sreenivasan 1982; Piomelli \& Yuan 2013). Figure 21(b) however shows a contrasting picture. In most cases (except for D1 and B21), the distributions of the streamwise vorticity in the body-force-influenced cases agree reasonably well with their respective EPG reference flows. This implies that the imposition of the body forces does not seem to cause any significant changes in the generation of streamwise vorticity, an important stage in the turbulence regeneration cycle. The increase of the vorticity fluctuations in B21 and D1 can be related to fact that the body-force-generated turbulent shear stress is larger in those cases, which apparently causes some increase in streamwise vorticity activities.

Figure 19 shows that the streaks are strengthened by the imposition of the body force. To further characterise the streaks, figure 22 shows the contours of the spanwise 


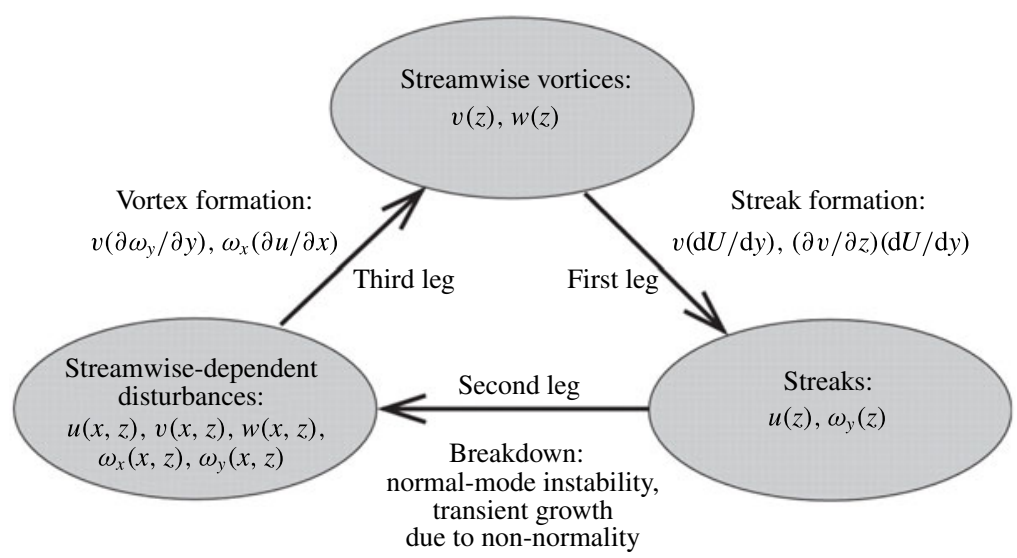

FIGURE 20. Schematic of a self-sustaining process of near-wall turbulence structures. (Reproduced from Kim 2011 with permission.)
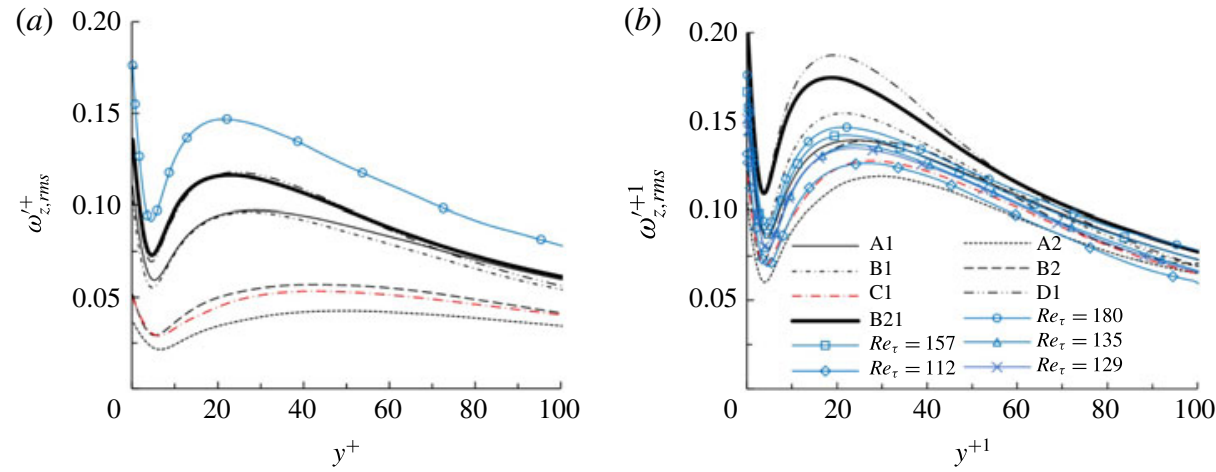

FIGURE 21. (Colour online) Root-mean-square of streamwise vorticity fluctuations normalised using the $(a)$ conventional friction velocity $(b)$ apparent friction velocity.

correlations of the streamwise velocity on the $y-r \theta$ plane in wall coordinates based on the total flow. The data plotted are the amplitude of the negative correlations with the positive correlations set to zero. The locations of the peak values (i.e. minimum correlation) provide the following information: $2 \times(r \theta)_{\min }$ is the averaged spacing of the steaks and $y_{\min }$ is the averaged wall distance from the centre of the streaks. It can be seen from figure 22 that the averaged spacing of the streaks in the base flow is around $110\left((r \theta)_{\min }^{+}=55\right)$ and the averaged wall distance of the streaks is $y_{\min }^{+}=14$. The streaks spacing in most of the test cases is increased and in some cases, significantly. The largest spacing, occurring in A2, is around $(r \theta)^{+}=170$. The streaks' centre also moves away from the wall, and the largest distance is around $y^{+}=20$, occurring in A2 and B2. There are some exceptions to the trends described above; for example, both the streaks spacing and the location do not change significantly in B21 and D1. The same data shown in the above figure are shown again in figure 23 in the $y-r \theta$ plane in wall coordinates based on the apparent friction velocity. In most cases, both the averaged spacing of the steaks $\left(2 \times(r \theta)_{\min }\right)$ and the wall distance of the streaks centres are now similar to those of the base flow. The exceptions are cases B2, D1 and B21, where the spacing is reduced to approximately 90, but the 

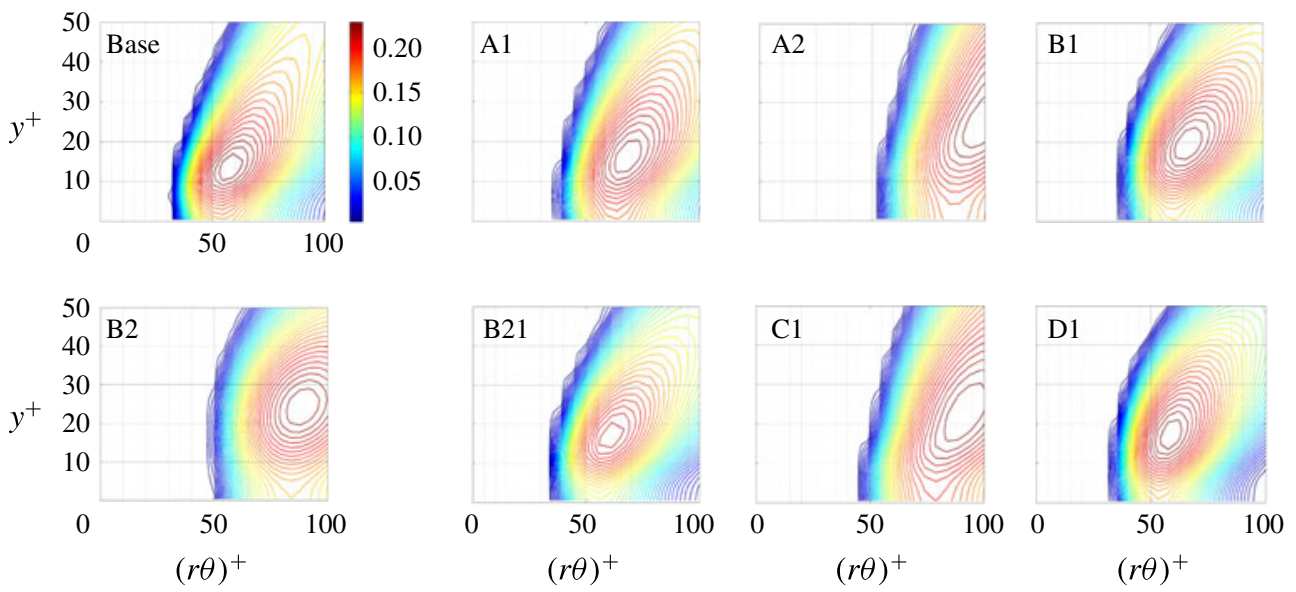

FIGURE 22. (Colour online) Contours of the streamwise velocity correlations in a vertical plane in wall units based on the conventional friction velocity. The values of the contours reduce monotonically from the outer edge of the contours to the centre.
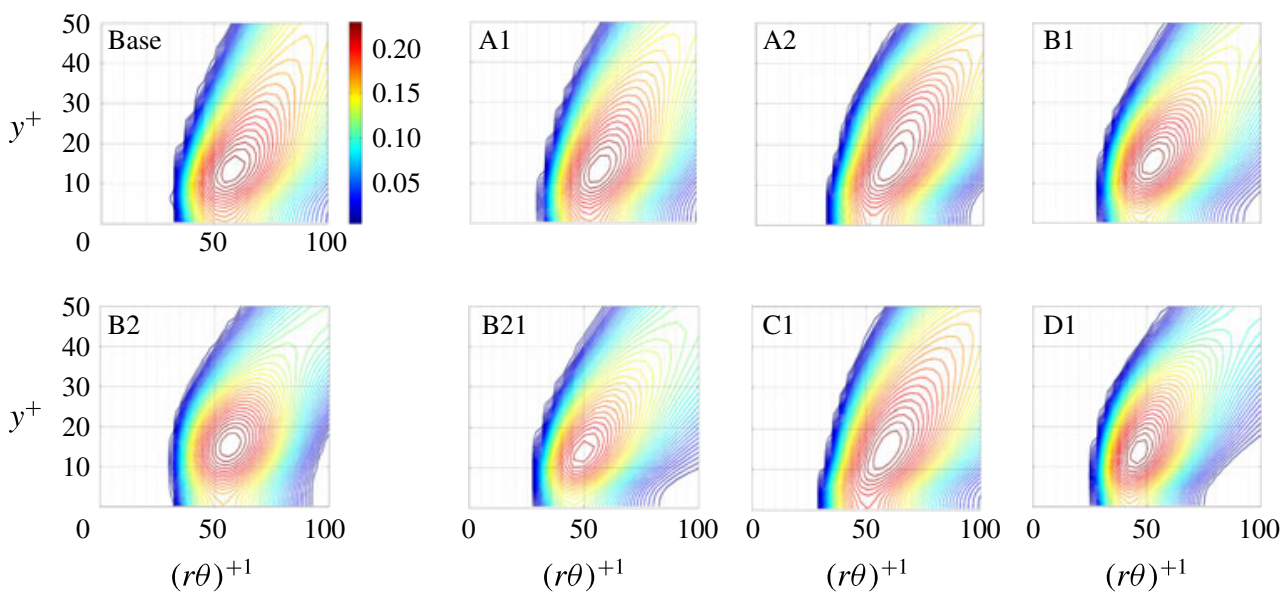

Figure 23. (Colour online) Contours of the streamwise velocity correlations in a vertical plane in wall units based on an apparent friction velocity. The values of the contours reduce monotonically from the outer edge of the contours to the centre.

vertical locations of the streaks centres are still similar to that of the reference flow. As observed earlier, these are the cases where the turbulence deviates somewhat from their corresponding EPG reference flows.

The near-wall turbulence structures can be studied through inspecting the ejection and sweep events which are associated with the vortices and streaks (Kline et al. 1967; Wallace, Eckelmann \& Brodkey 1972; Kim et al. 1987; Robinson 1991). Using the definition of the hyperbolic hole introduced by $\mathrm{Lu} \&$ Willmarth (1973), the contribution of each quadrant to $\overline{u_{z}^{\prime} u_{r}^{\prime}}$ is defined as

$$
\left(\overline{u_{z}^{\prime} u_{r}^{\prime}}\right)_{Q}=\lim _{T \rightarrow \infty} \frac{1}{T} \int_{0}^{T}\left(u_{z}^{\prime} u_{r}^{\prime}\right) I(t) \mathrm{d} t
$$



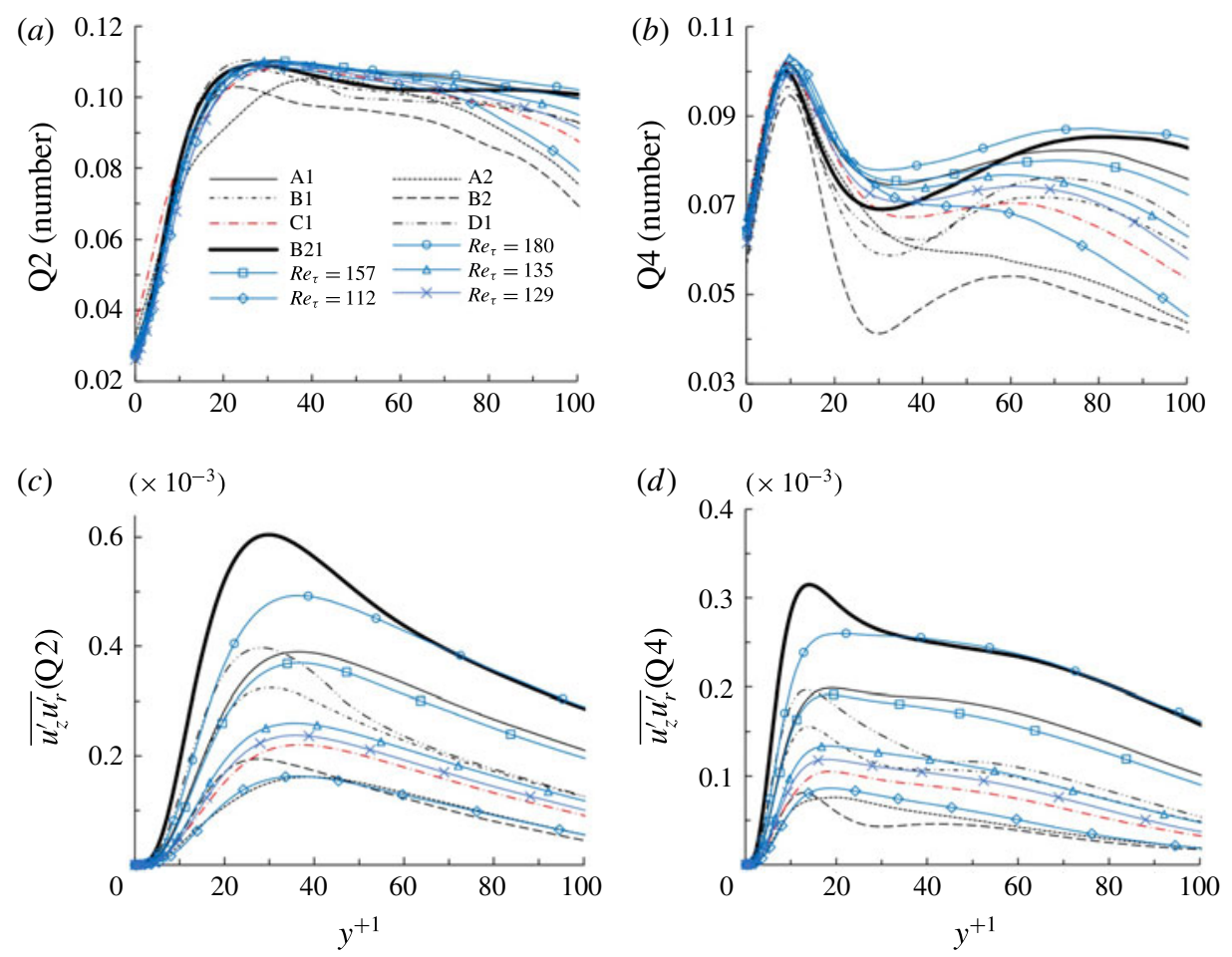

FIgURE 24. (Colour online) Quadrant analysis. (a) Number of ejections (Q2) over the total number of events; $(b)$ number of sweeps (Q4) over the total number of events; (c) contributions of ejection (Q2) events to $\overline{u_{z}^{\prime} u_{r}^{\prime}}$; $(d)$ contributions of sweep (Q4) events to $\overline{u_{z}^{\prime} u_{r}^{\prime}}$.

and the percentage number count is

$$
N_{Q}=\lim _{T \rightarrow \infty} \frac{1}{T} \int_{0}^{T} I(t) \mathrm{d} t,
$$

where $Q=1, \ldots, 4$ is the quadrant and

$$
I(t)= \begin{cases}1, & \text { if }\left|u_{z}^{\prime} u_{r}^{\prime}\right|_{Q} \geqslant h u_{z, r m s}^{\prime} u_{r, r m s}^{\prime} \\ 0, & \text { otherwise. }\end{cases}
$$

The greater the value of $h$, the stronger the events are. Figure 24(a,b) show the percentages of the numbers of ejections and sweeps of the total turbulent events that result in turbulent shear stress, and figure $24(c, d)$ show the values of the shear stress associated with these two activities. A strength of $h=1$ is used. It is most interesting to see that the number of the events in the body-force-influenced cases remains more or less unchanged from the base flow distribution. For ejections the agreement between them is very good, within a few per cent, whereas for sweeps the deviations are slightly larger but are still within approximately $10 \%$ for most cases. The only exception is B2 where the number is significantly lower than in all other cases. The contributions to shear stress from the ejections and sweeps also remain largely unchanged in the low $\left(\overline{u_{z}^{\prime} u_{r}^{\prime}}\right)_{b}$ flows, but they are significantly 
increased in the wall region in other cases as a result of the imposition of the body force. That is, the strength of each event in these cases is greater. This can be nicely linked to the observations discussed above on vorticity and streaks. It is apparent that the imposition of the body force, which induces an additional boundary layer near the wall, does not significantly influence the generation of vortices, but for each ejection or sweep event, a larger shear stress is resulted in due to the larger velocity gradient. Additionally it is interesting to note that the influence on the ejection events occurs in the region of $y^{+1}<60$, where the effect on sweep is restricted to $y^{+1}<30$.

Finally, we evaluate the turbulence anisotropy by analysing the anisotropy-invariant map (AIM) of the Reynolds stress anisotropy tensor $\left(b_{i j}\right)$ :

$$
b_{i j}=\frac{\overline{u_{i} v_{j}}}{\overline{u_{k} u_{k}}}-\frac{\delta_{i j}}{3},
$$

where $\boldsymbol{\delta}_{i j}$ is the Kronecker delta tensor. The AIM proposed by Lumley (1978) is constructed using the second and third principal invariants $\left(I I=-(1 / 2)\left(b_{z z}^{2}+b_{r r}^{2}+\right.\right.$ $\left.b_{\theta \theta}^{2}+2 b_{z r}^{2}\right)$ and $\left.I I I=\left(b_{\theta \theta} b_{z z} b_{r r}+b_{\theta \theta} b_{z r}^{2}\right)\right)$, which are independent of the coordinate choice. Lumley's anisotropic map provides useful information on the streaky structures since they are inherently anisotropic. The AIMs for the various body-force-influenced cases are plotted on top of that of the EFR reference flow in figure 25. It can be seen clearly that in all cases the profile moves towards the top right vertex, indicating that the flow becomes more one-dimensional and further demonstrating the streaky structures are elongated under the influence of the body force. The stronger the body force, the stronger the one-dimensional feature of the flow. In fact, the profile nearly reaches the top-right vertex in A2 and B2, showing the existence of strong streaks in these flows (also refer to figure 19 to see the streaks in B2). The profile in the undisturbed turbulent flow often reaches the topmost point at around $y^{+}=9$. It can be seen that this point is only shifted slightly away from the wall in the body-force-influenced cases even though the strength are significantly increased. When measured using the wall units based on the apparent friction velocity, the location actually lowers down slightly.

\subsection{Applications and limitations}

The present study is based on two types of idealised body-force distributions, i.e. linear and step changes with a systematic variation of the coverage and amplitude of the body forces. Strictly speaking, the conclusions drawn herein are only directly applicable to such situations. However, the principle conclusions stem from an observation that the non-uniform body forces, whether linear or step change, do not alter the turbulence mixing characteristics in any significant way. By nature, such conclusions are not dependent on the detailed profile of the body force, and are likely to be applicable to more complex distributions. Nevertheless, care should be taken when extrapolating the conclusions to new situations, and in particular, when the body force extends further away from the wall than considered herein.

The understanding developed herein potentially provides new insight into the laminarisation of flow due to buoyant forces in mixed convection in vertical channels. However, it should be noted that, in practice, the flow and thermal fields are closely coupled in mixed convection, and both fields may continue developing streamwise due to the temperature dependence of fluid properties. Such effects are not addressed herein. It would naturally be a useful and interesting development next to analyse mixed convection with coupled flow and thermal fields to understand the full picture of the physical problem. 
(a)
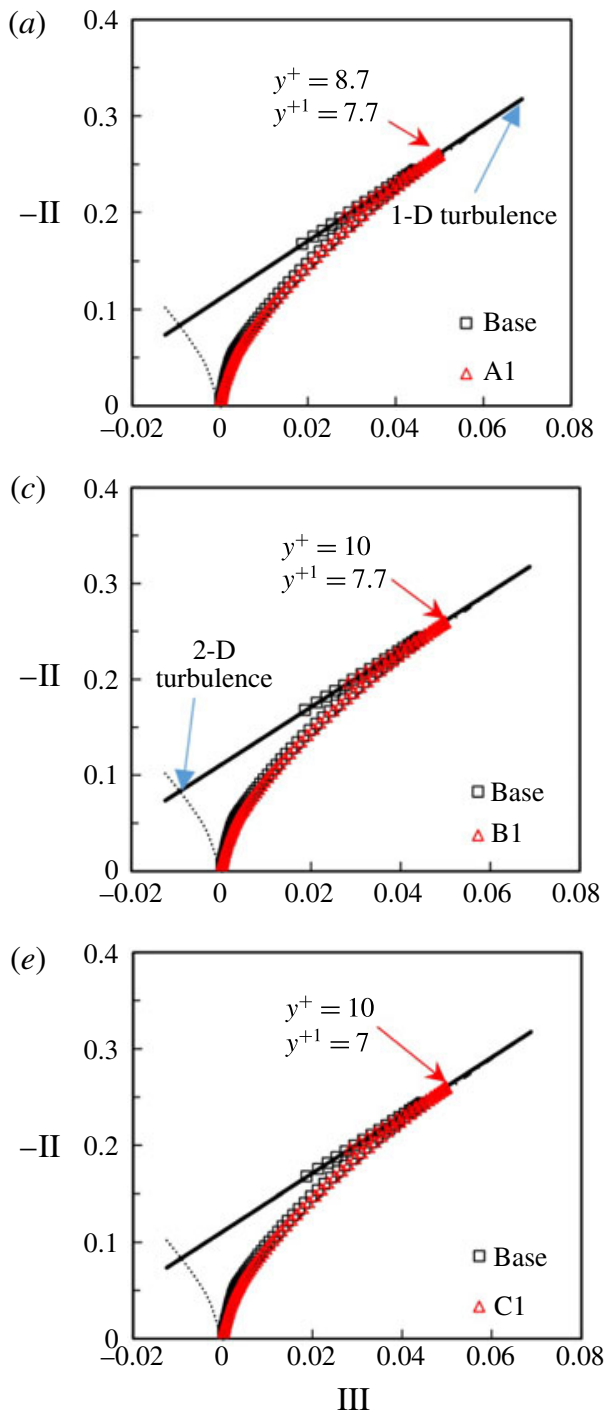

(b)

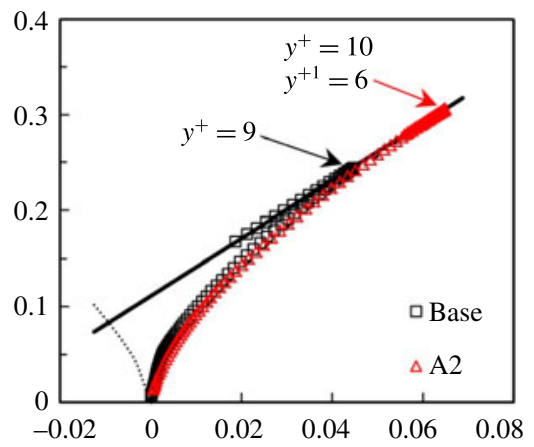

(d)

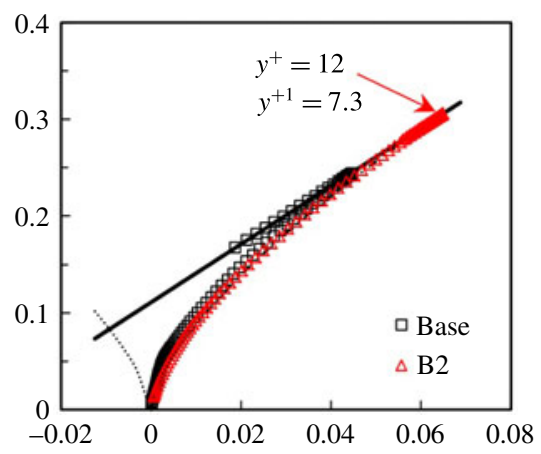

$(f)$

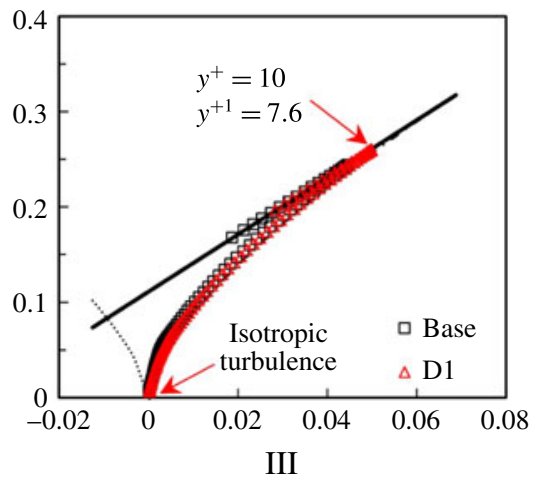

FIgURE 25. (Colour online) The anisotropy-invariant maps (AIMs) of a number of flow cases.

\section{Summary and conclusions}

(i) The conventional viewpoint of flow laminarisation. When a turbulent flow is subjected to a non-uniform streamwise body force in the same direction of the flow (body-force-aided flow), the turbulence may be significantly reduced in comparison with the flow of the same Reynolds number but without the body force. Under such a situation, the flow is said to be laminarised. In general, the stronger the body force, the stronger the reduction of the turbulence is. When the body force is sufficiently strong, the flow may be fully laminarised. This trend has been observed in the literature for various physical flows, including, for example, the buoyancy-aided mixed convection when severe heat transfer deterioration may occur. In the present study, DNS has been performed for 
a turbulent flow in a pipe subjected to two typical distributions of body force: a linear distribution and a stepwise variation. It has been found that both types of body force can effectively laminarise the flow, reproducing the phenomena that have been observed in previous studies. A comprehensive picture on the effects of the profile, amplitude and spatial coverage of the body force on the flow and turbulence has been established by systematically varying the distribution of the body force. In addition to achieving a better understanding of flow physics, the results also supplement the existing data of physical flows, enabling further validation and development of turbulence models.

(ii) New insights into the influence of non-uniform body force on turbulence. The body-force-aided flow can be interpreted as resulting from imposing a non-uniform body force to a turbulent flow initially driven by a pressure force only. We refer to the latter to be the EPG reference flow of the former; they share the same pressure gradient. The following are demonstrated to be true:

(a) The key characteristics of the turbulence of the flow remains largely unchanged following the imposition of the body force. Most significantly the turbulent viscosity of the flow remains largely uninfluenced by the imposition of the body force, even though the total flow rate can be significantly increased.

(b) The wall-normal and circumferential turbulent stresses remain largely unchanged, but the streamwise turbulent stress can be increased. The latter is a result of enhanced high- and low-speed streaks.

(c) In terms of the turbulence regeneration cycle, the numbers of ejections and sweeps are little influenced by the imposition of the body force, whereas the strength of each event may/may not be stronger dependent on the distribution of the body force. The former is true when the coverage of the body force extends significantly away from the wall, and under such a condition, the body-force-induced flow perturbation results in a greater turbulent shear stress.

(d) The perturbation flow caused by the imposition of the body force is estimated by solving the following momentum equation,

$$
0=\frac{1}{r} \frac{\partial}{\partial r}\left[\frac{r}{R e}\left(\left(1+v_{t 1}\right) \frac{\partial\left(\overline{u_{z}}\right)_{b}}{\partial r}\right)\right]+f,
$$

where $v_{t 1}$ is the turbulent viscosity of the EPG reference flow and therefore can be seen as known. The velocity field can be obtained simply from the integration of the above equation.

(e) The wall shear of the body-force-influenced flow can be closely approximated by the sum of the wall shear of the EPG reference flow and that of the body-force-driven flow obtainable from the above momentum equation.

( $f$ ) When the coverage of the body force is small, say, less than $y^{+}<20$, the body-force-induced perturbation flow is effectively a laminar flow. The above equation is further simplified with the dropping of $v_{t 1}$.

(iii) A new perspective on flow laminarisation. The new insights into the effect of body force on turbulence detailed above afford new interpretations for the flow laminarisation such as those observed in buoyancy-influenced mixed convection. 
(a) Imposing a body force to a turbulent flow while keeping the total flow rate the same, in effect, causes a reduction in the apparent Reynolds number of the flow based on an apparent friction velocity associated with the pressure force of the flow (i.e. excluding the component due to the body force). The stronger the body force, the lower the apparent Reynolds number, and hence the lower the turbulence. In comparison with the flow of the same flow rate, the flow is more strongly laminarised.

(b) The strength of the effect of a body force on the flow in terms of 'suppressing' turbulence and 'laminarising' the flow is predominately associated with the flow rate of the perturbation flow it induces. The apparent flow (as far as the turbulence is concerned) is the total flow minus the perturbation flow of the body force. Hence, the higher the latter, the smaller the apparent flow; the lower the apparent Reynolds number; and the stronger the laminarisation. The flow rate of the perturbation flow is linearly proportional to the amplitude of the body-force distribution. It also increases with the increase of the coverage when the amplitude is fixed and the relation is described by (3.12).

(c) The profile of the body-force distribution affects the laminarisation mostly through the flow rate that it causes. For example, with the same amplitude and coverage, the stepwise distribution causes a perturbation flow that is approximately 2.6 to 3 times higher than that of a linear distribution. Considering the ratio between the total forces of the stepwise and linear distributions of the same amplitude and coverage is approximately 2, the step distribution is therefore more effective in 'laminarising' the flow.

(d) When the applied body force is sufficiently large, causing the apparent Reynolds number of the flow to be sufficiently small and turbulence unsustainable, the flow is fully laminarised. For any given body force, the critical Reynolds number can be calculated with the knowledge of 'undisturbed' turbulent flow.

\section{Acknowledgements}

We gratefully acknowledge that the work reported herein is partially funded by UK Engineering and Physical Science Research Council (grant no. EP/G068925/1). K.H. is jointly sponsored by Chinese Scholarship Council and the University of Sheffield. Some earlier work was carried out making use of the UK national supercomputer ARCHER, access to which was provided by UK Turbulence Consortium funded by the Research Council (grant no. EP/L000261/1).

\section{REFERENCES}

Abdelmeguid, A. M. \& Spalding, D. B. 1979 Turbulent-flow and heat-transfer in pipes with buoyancy effects. J. Fluid Mech. 94, 383-400.

Aicher, T. \& MARTin, H. 1997 New correlations for mixed turbulent natural and forced convection heat transfer in vertical tubes. Intl J. Heat Mass Transfer 40 (15), 3617-3626.

BAE, J. H., Yoo, J. Y. \& CHOI, H. 2005 Direct numerical simulation of turbulent supercritical flows with heat transfer. Phys. Fluids 17 (10), 105104.

Bae, J. H., Yoo, J. Y., Choi, H. \& Mceligot, D. M. 2006 Effects of large density variation on strongly heated internal air flows. Phys. Fluids 18 (7), 075102. 
Bae, J. H., Yoo, J. Y. \& Mceligot, D. M. 2008 Direct numerical simulation of heated $\mathrm{CO}_{2}$ flows at supercritical pressure in a vertical annulus at $R e=8900$. Phys. Fluids 20 (5), 055108.

CArr, A. D., Connor, M. A. \& Buhr, H. O. 1973 Velocity, temperature, and turbulence measurements in air for pipe-flow with combined free and forced convection. Trans. ASME J. Heat Transfer 95 (4), 445-452.

Celata, G. P., D’ Annibale, F., Chiaradia, A. \& Cumo, M. 1998 Upflow turbulent mixed convection heat transfer in vertical pipes. Intl J. Heat Mass Transfer 41 (24), 4037-4054.

CotTon, M. A. \& JACKSON, J. D. 1990 Vertical tube air-flows in the turbulent mixed convection regime calculated using a low-Reynolds-number k-epsilon model. Intl J. Heat Mass Transfer 33 (2), 275-286.

Craft, T. J., Gant, S. E., Iacovides, H. \& Launder, B. E. 2004 A new wall function strategy for complex turbulent flows. Numer. Heat Transfer B 45 (4), 301-318.

Crawford, C. H. \& Karniadakis, G. E. 1997 Reynolds stress analysis of EMHD-controlled wall turbulence. 1. Streamwise forcing. Phys. Fluids 9 (3), 788-806.

Escudier, M. P., Abdel-Hameed, A., Johnson, M. W. \& Sutcliffe, C. J. 1998 Laminarisation and re-transition of a turbulent boundary layer subjected to favourable pressure gradient. Exp. Fluids 25 (5-6), 491-502.

Fukagata, K., Iwamoto, K. \& Kasagi, N. 2002 Contribution of Reynolds stress distribution to the skin friction in wall-bounded flows. Phys. Fluids 14 (11), L73-L76.

Greenblatt, D. \& Moss, E. A. 2004 Rapid temporal acceleration of a turbulent pipe flow. J. Fluid Mech. 514, 65-75.

He, K., Seddighi, M. \& He, S. 2016 DNS study of a pipe flow following a step increase in flow rate. Intl J. Heat Fluid Flow 57, 130-141.

HE, S., KIM, W. S. \& BAE, J. H. 2008 Assessment of performance of turbulence models in predicting supercritical pressure heat transfer in a vertical tube. Intl J. Heat Mass Transfer 51 (19), $4659-4675$.

He, S. \& Seddighi, M. 2013 Turbulence in transient channel flow. J. Fluid Mech. 715, 60-102.

JACKSON, J. D. 2013 Fluid flow and convective heat transfer to fluids at supercritical pressure. Nucl. Engng Des. 264, 24-40.

Jackson, J. D., Cotton, M. A. \& AxCell, B. P. 1989 Studies of mixed convection in vertical tubes. Intl J. Heat Fluid Flow 10 (1), 2-15.

JACKSON, J. D. \& HALL, W. B. 1979 Influences of buoyancy on heat transfer to fluids flowing in vertical tubes under turbulent conditions. In Turbulent Forced Convection in Channels and Bundles Theory and Applications to Heat Exchangers and Nuclear Reactors, 2, Advanced Study Institute Book (ed. S. Kakac \& D. B. Spalding), pp. 613-640. Hemisphere.

JiAnG, P. X., SHI, R. F., XU, Y. J., HE, S. \& JACKSON, J. D. 2006 Experimental investigation of flow resistance and convection heat transfer of $\mathrm{CO}_{2}$ at supercritical pressures in a vertical porous tube. J. Supercritical Fluids 38 (3), 339-346.

KASAgi, N. \& NishimuRA, M. 1997 Direct numerical simulation of combined forced and natural turbulent convection in a vertical plane channel. Intl J. Heat Fluid Flow 18 (1), 88-99.

Keshmiri, A., Cotton, M. A., Addad, Y. \& Laurence, D. 2012 Turbulence models and large eddy simulations applied to ascending mixed convection flows. Flow Turbul. Combust. 89 (3), 407-434.

KIM, J. 2011 Physics and control of wall turbulence for drag reduction. Phil. Trans. R. Soc. Lond. A 369 (1940), 1396-1411.

Kim, J., Moin, P. \& Moser, R. 1987 Turbulence statistics in fully developed channel flow at low Reynolds number. J. Fluid Mech. 177, 133-166.

KIM, W. S., HE, S. \& JACKSON, J. D. 2008 Assessment by comparison with DNS data of turbulence models used in simulations of mixed convection. Intl J. Heat Mass Transfer 51 (5-6), 1293-1312.

Kline, S. J., Reynolds, W. C., Schraub, F. A. \& Runstadler, P. W. 1967 The structure of turbulent boundary layers. J. Fluid Mech. 30 (04), 741-773.

Kurganov, V. A. \& Kaptilny, A. G. 1992 Velocity and enthalpy fields and eddy diffusivities in a heated supercritical fluid-flow. Exp. Therm. Fluid Sci. 5 (4), 465-478. 
Launder, B. E. \& Jones, W. P. 1969 Sink flow turbulent boundary layers. J. Fluid Mech. 38, $817-831$.

LEE, D. \& CHOI, H. 2001 Magnetohydrodynamic turbulent flow in a channel at low magnetic Reynolds number. J. Fluid Mech. 439, 367-394.

Lee, J. I., Hejzlar, P., Saha, P., Kazimi, M. S. \& Mceligot, D. M. 2008 Deteriorated turbulent heat transfer (DTHT) of gas up-flow in a circular tube: heat transfer correlations. Intl J. Heat Mass Transfer 51 (21-22), 5318-5326.

LiaO, S. M. \& ZHAO, T. S. 2002 An experimental investigation of convection heat transfer to supercritical carbon dioxide in miniature tubes. Intl J. Heat Mass Transfer 45 (25), 5025-5034.

Licht, J., Anderson, M. \& Corradini, M. 2009 Heat transfer and fluid flow characteristics in supercritical pressure water. Trans. ASME J. Heat Transfer 131 (7), 072502.

LU, S. S. \& Willmarth, W. W. 1973 Measurements of the structure of the Reynolds stress in a turbulent boundary layer. J. Fluid Mech. 60 (03), 481-511.

Lumley, J. L. 1978 Computational modeling of turbulent flows. Adv. Appl. Mech. 18 (123), 213.

Mikielewicz, D. P., Shehata, A. M., Jackson, J. D. \& Mceligot, D. M. 2002 Temperature, velocity and mean turbulence structure in strongly heated internal gas flows - comparison of numerical predictions with data. Intl J. Heat Mass Transfer 45 (21), 4333-4352.

Nakajima, M., Fukui, K., Ueda, H. \& Mizushina, T. 1980 Buoyancy effects on turbulent transport in combined free and forced-convection between vertical parallel plates. Intl J. Heat Mass Transfer 23 (10), 1325-1336.

Orlandi, P. 2000 Fluid Flow Phenomena - A Numerical Toolkit. Fluid Mechanics and Its Applications, vol. 55. Springer.

Petukhov, B. S., Polyakov, A. F. \& Launder, B. E. 1988 Heat Transfer in Turbulent Mixed Convection. Hemisphere.

Piomelli, U., Balaras, E. \& Pascarelli, A. 2000 Turbulent structures in accelerating boundary layers. J. Turbul. 1 (1), N1.

PiomelLI, U.\& YUAN, J. 2013 Numerical simulations of spatially developing, accelerating boundary layers. Phys. Fluids 25 (10), 101304.

Polyakov, A. F. \& Shindin, S. A. 1988 Development of turbulent heat-transfer over the length of vertical tubes in the presence of mixed air convection. Intl J. Heat Mass Transfer 31 (5), 987-992.

Robinson, S. K. 1991 Coherent motions in the turbulent boundary layer. Annu. Rev. Fluid Mech. 23 (1), 601-639.

Satake, S., Kunugi, T., Shehata, A. M. \& Mceligot, D. M. 2000 Direct numerical simulation for laminarization of turbulent forced gas flows in circular tubes with strong heating. Intl $J$. Heat Fluid Flow 21 (5), 526-534.

SEDdighi, M. 2011 Study of turbulence and wall shear stress in unsteady flow over smooth and rough wall surfaces. PhD thesis, University of Aberdeen.

Shehata, A. M. \& Mceligot, D. M. 1998 Mean structure in the viscous layer of strongly-heated internal gas flows. measurements. Intl J. Heat Mass Transfer 41 (24), 4297-4313.

SREENIVASAN, K. R. 1982 Laminarescent, relaminarizing and retransitional flows. Acta Mechanica $44(1-2), 1-48$.

STEINER, A. 1971 Reverse transition of a turbulent flow under action of buoyancy forces. J. Fluid Mech. 47, 503-512.

Sumitani, Y. \& KaSAgi, N. 1995 Direct numerical-simulation of turbulent transport with uniform wall injection and suction. AIAA J. 33 (7), 1220-1228.

Tsukahara, T., Seki, Y., Kawamura, H. \& Tochio, D. 2014 DNS of turbulent channel flow at very low Reynolds numbers. arXiv:1406.0248.

Vilemas, J. V., POSKAS, P. S. \& KaUPAS, V. E. 1992 Local heat-transfer in a vertical gas-cooled tube with turbulent mixed convection and different heat fluxes. Intl J. Heat Mass Transfer 35 (10), 2421-2428.

Wallace, J. M, Eckelmann, H. \& Brodkey, R. S. 1972 The wall region in turbulent shear flow. J. Fluid Mech. 54 (01), 39-48. 
WANG, J. L., LI, J. K. \& JACKSON, J. D. 2004 A study of the influence of buoyancy on turbulent flow in a vertical plane passage. Intl J. Heat Fluid Flow 25 (3), 420-430.

WU, X. \& MoIN, P. 2008 A direct numerical simulation study on the mean velocity characteristics in turbulent pipe flow. J. Fluid Mech. 608, 81-112.

Xu, J., Dong, S., MaXey, M. R. \& Karniadakis, G. E. 2007 Turbulent drag reduction by constant near-wall forcing. J. Fluid Mech. 582, 79-101.

Yoo, J. Y. 2013 The turbulent flows of supercritical fluids with heat transfer. Annu. Rev. Fluid Mech. 45, 495-525.

You, J., Yoo, J. Y. \& ChOI, H. 2003 Direct numerical simulation of heated vertical air flows in fully developed turbulent mixed convection. Intl J. Heat Mass Transfer 46 (9), 1613-1627. 